\title{
A stochastic fractional dynamics model of space-time variability of rain
}

\author{
Prasun K. Kundu ${ }^{1,2}$ and James E. Travis ${ }^{3}$ \\ Received 17 December 2012; revised 7 August 2013; accepted 7 August 2013; published 18 September 2013.
}

[1] Rainfall varies in space and time in a highly irregular manner and is described naturally in terms of a stochastic process. A characteristic feature of rainfall statistics is that they depend strongly on the space-time scales over which rain data are averaged. A spectral model of precipitation has been developed based on a stochastic differential equation of fractional order for the point rain rate, which allows a concise description of the second moment statistics of rain at any prescribed space-time averaging scale. The model is thus capable of providing a unified description of the statistics of both radar and rain gauge data. The underlying dynamical equation can be expressed in terms of space-time derivatives of fractional orders that are adjusted together with other model parameters to fit the data. The form of the resulting spectrum gives the model adequate flexibility to capture the subtle interplay between the spatial and temporal scales of variability of rain but strongly constrains the predicted statistical behavior as a function of the averaging length and time scales. We test the model with radar and gauge data collected contemporaneously at the NASA TRMM ground validation sites located near Melbourne, Florida and on the Kwajalein Atoll, Marshall Islands in the tropical Pacific. We estimate the parameters by tuning them to fit the second moment statistics of radar data at the smaller spatiotemporal scales. The model predictions are then found to fit the second moment statistics of the gauge data reasonably well at these scales without any further adjustment.

Citation: Kundu, P. K., and J. E. Travis (2013), A stochastic fractional dynamics model of space-time variability of rain, J. Geophys. Res. Atmos., 118, 10,277-10,295, doi:10.1002/jgrd.50723.

\section{Introduction}

[2] Because of its irregular nature, rain is both difficult to measure accurately and predict from a physical model. Models of rain statistics provide a simple and conceptually economical way to capture the space-time variability of precipitation in terms of a small number of adjustable parameters. They can be relatively easily validated from a large space-time data set, and once the parameters are tuned to data, the model provides a rather efficient method of describing various statistical properties of precipitation over areas of similar rain climatologies.

[3] In practice, rainfall is generally measured as a (nearly) instantaneous area-averaged quantity in radar measurements or as a time-averaged quantity at a point in rain gauge measurements. In theoretical models, they are

\footnotetext{
${ }^{1}$ Joint Center for Earth Systems Technology, University of Maryland, Baltimore County, Baltimore, Maryland, USA.

${ }^{2}$ Joint Center for Earth Systems Technology, NASA Goddard Space Flight Center, Greenbelt, Maryland, USA.

${ }^{3}$ Department of Mathematics and Statistics, University of Maryland, Baltimore County, Baltimore, Maryland, USA.

Corresponding author: P. K. Kundu, Joint Center for Earth Systems Technology, University of Maryland, Baltimore County, 5523 Research Park Drive, Baltimore, MD 21228, USA. (prasun.k.kundu@nasa.gov)

C2013. American Geophysical Union. All Rights Reserved. 2169-897X/13/10.1002/jgrd.50723
}

conveniently represented as suitable space and/or time averages of a continuous stochastic field. This continuum approximation is valid at the space-time resolution of the usual measurement methods under normal rainy conditions in which the inherent discreteness of rain at the scale of individual drops is smoothed out. In this paper we develop a phenomenological model of space-time statistics of rain in terms of a random field $R(\mathbf{x}, t)$ denoting the instantaneous point rain rate. It should be emphasized that $R(\mathbf{x}, t)$ is not directly observable, but when suitably area- or time-averaged, corresponds to measured quantities.

[4] Radar scans yield area averages at an instant of time with horizontal spatial resolution of order $1 \mathrm{~km}$. Rain gauge and disdrometer observations, on the other hand, lead to time-averaged point rain rate estimates with temporal resolution of order $1 \mathrm{~min}$. These data can then be further "coarse-grained" by aggregating them to any desired larger space-time scale. Although rainfall varies in an apparently irregular manner, the underlying physical processes take place over an extended space-time region that causes the rain rate field to be correlated in space and time. Moreover, the statistics of rainfall depend on the space-time averaging scales in a nontrivial manner. In fact, it is well-known that [see, e.g., Bell, 1987] there is a subtle interplay between the space-time scales associated with the decay of the correlation function and the averaging scale that is reminiscent of fluid turbulence. Like the velocity field of a turbulent fluid, the rain rate field $R(\mathbf{x}, t)$ has the property that the larger the 
averaging area, the longer the field remains temporally correlated. Similarly, the longer the period of time averaging, the greater the distance over which the spatial correlation persists. This property of the space-time correlation of rain is most easily captured in terms of the Fourier spectrum of the field. A spectral model of precipitation statistics was developed in a number of earlier papers [Bell and Kundu, 1996] (hereinafter BK96) and [Kundu and Bell, 2003] (hereinafter $\mathrm{KB} 03$ ) that incorporates these features in a qualitative manner. The spectrum is generated from a Langevin-type stochastic differential equation for the spatial Fourier amplitudes of $R(\mathbf{x}, t)$ that is suggested by analogy with Brownian motion. The model spectrum in turn directly determines the complete second moment statistics of the rain field averaged to any desired space-time scale and is thus in principle capable of fitting both radar and rain gauge data. Thus, if the parameters of the model are tuned to fit the statistics of area-averaged rain rate using radar rainfall data, the model is then expected to describe, without any further adjustment, the statistics of time-averaged point rain rate data from a gauge network within the same space-time domain. We refer the reader to BK96 and KB03 for a more complete account of various aspects of the model and the relevant literature on other modeling approaches.

[5] With the availability of large precipitation data sets in recent years, it has become feasible to validate the model quantitatively over a large range of space-time scales. Large multiyear data sets have now been produced from ongoing radar and rain gauge measurements collected as part of the ground validation $(\mathrm{GV})$ program pursued by NASA during the Tropical Rainfall Measuring Mission (TRMM) [Wolff et al., 2005]. In particular, a large amount of space-time colocated data is available from radar and gauge observations at several TRMM GV sites. We sought to test the spectral model described in BK96 and KB03 with the TRMM GV data. During this effort, it became clear that the model in its original form broadly captures the general features of the space-time statistics of radar-derived precipitation data but does not accurately fit the details. Moreover, if the model parameters are estimated by fitting the radar data, the predicted statistics of the accompanying rain gauge data depart substantially from the observed statistics. Alternatively, the parameters estimated independently from the radar and gauge observations belong to qualitatively distinct model regimes. The inevitable conclusion was that the originally proposed model spectrum needed to be generalized for it to describe both radar and gauge observations.

[6] Our present work stems from an attempt to find such a generalization. With the integration of radar and gauge observations, a larger range of space-time scales becomes experimentally accessible. In order to achieve greater flexibility in fitting all the available data, we extend the model framework by generalizing the underlying stochastic dynamical equation from an ordinary differential equation in time to a differential equation of a suitable noninteger order. The new model is able to fit the second moment statistics of both radar and gauge data more closely than the original model over the accessible range of space-time scales. It should be emphasized that the model describes only the second moment statistics of $R(\mathbf{x}, t)$, not the full probability distribution which is also known to depend on the space-time averaging scale [Kedem and Chiu, 1987; Bell, 1987; Kundu and Siddani, 2007].
[7] The stochastic equation introduced in this paper to describe the precipitation process involves a mathematical framework generally referred to as fractional calculus. Broadly speaking, fractional calculus constitutes an extension of the notion of derivatives and integrals of ordinary calculus to derivatives and integrals of fractional order [Miller and Ross, 1993; Oldham and Spanier, 2006; Samko et al., 1993]. West et al. [2003] has given a thought-provoking account of how such fractional operators can arise in the description of a wide variety of macroscopic physical processes. While these fractional differential operators can be mathematically formulated in several different ways, their representation, as certain integral operators with a power law kernel known as Riemann-Liouville operators, lends itself to the clearest physical interpretation. The new model generalizes the "old" spectral model of BK96 and KB03 by replacing the ordinary time derivative in the underlying stochastic dynamical equation by a fractional derivative operator. Because of the postulated power law dependence of the relaxation time of the Fourier modes in BK96 and KB03, the fluctuations of the rain field were already implicitly nonlocal in space. Now the nonlocality in time evolution implied by the power law kernel of the fractional time derivative operator also reflects the presence of a memory [Beran, 1994].

[8] One noticeable shortcoming of the original BK96 model with the time evolution governed by a first-order time derivative was that the model did not fit the lagged autocorrelation function of the area-averaged rain rate very well. The falloff rate of the lagged autocorrelation with lag $\tau$ predicted by the model was found to differ markedly from what was actually observed, especially for small $\tau$, and it was suggested in BK96 that this indicated the need for introducing higher order autoregressive processes. The introduction of a fractional-order time derivative allows us to control the shape at small $\tau$ effectively in a parsimonious manner and thereby obtain much better fit to the observed lagged autocorrelation in this regime.

[9] We have tested the validity of our model using two radar data sets belonging to TRMM standard product $2 \mathrm{~A}-53$ that were generated as part of the TRMM GV program: (i) a spatially gridded set of images generated from scans by a National Weather Service radar (hereafter called MELB) located near Melbourne, Florida and also (ii) a similar data set from the radar (hereafter called KWAJ) located on the Kwajalein Atoll, Republic of Marshall Islands in the Pacific Ocean. The MELB radar has the advantage that the portion of its field of view (FOV) that is over land contains a dense network of rain gauges. However, its coastal location creates a somewhat complicated precipitation climatology. On the other hand, the KWAJ radar FOV has the advantage of being in a predominantly oceanic environment. However, the few gauges that are available in the area are rather sparsely distributed. The gauge data used in this paper are part of TRMM standard product 2A-56.

[10] The remainder of the paper is organized as follows. In section 2, we give an account of the basic mathematical framework of the new stochastic model. In section 3, we first describe the radar data analysis. We then discuss the process of estimating the model parameters by fitting the model predictions to the observed second moment statistics of the MELB and KWAJ radar data. Finally, we test the model with the rain gauge data by examining how well the model tuned 
to the radar data reproduces the second moment statistics of rain data derived from a cluster of gauges located within the radar FOV. Section 4 is devoted to a discussion of the results along with the various caveats. The paper is concluded in section 5 with a summary of the findings and some directions for future work. In Appendix A we give a brief account of fractional calculus. Appendix B presents some details of the mathematical derivations of the necessary formulas and will be frequently referred to in the main text.

\section{The Model Framework}

[11] The Fourier spectrum of the precipitation field provides a convenient way to characterize the various aspects of its space-time variability in a succinct manner. In this section we first construct a stochastic dynamical model for the local rain rate field that naturally leads to such a spectrum. We then relate the space-time covariance statistics of the area- and timeaveraged rain rate to the spectrum through the Fourier transform representation. The resulting formulas are derived in Appendix B. In the last two subsections, we examine the behavior of the model in the limit of vanishingly small spacetime scales.

\subsection{The Basic Equations}

[12] In this subsection we describe the basic theoretical framework of the spectral model. A central quantity of interest is the space-time covariance of the point rain rate field $R(\mathbf{x}, t)$ at points $\mathbf{x}, \mathbf{x}^{\prime}$ in a two-dimensional Euclidean plane (neglecting the Earth's curvature) and at times $t, t^{\prime}$

$$
c\left(\mathbf{x}, t ; \mathbf{x}^{\prime}, t^{\prime}\right) \equiv\left\langle R^{\prime}(\mathbf{x}, t) R^{\prime}\left(\mathbf{x}^{\prime}, t^{\prime}\right)\right\rangle,
$$

where $R^{\prime}(\mathbf{x}, t)=R(\mathbf{x}, t)-\langle R\rangle$ is the deviation of the rain rate from the mean and the angle brackets $\langle\ldots\rangle$ denote ensemble average over similar rain climatologies. In our model it is determined from the Fourier spectrum of the rain field.

[13] As in BK96 and KB03, we assume the rain statistics to be spatially homogeneous, isotropic, and temporally stationary, (for brevity collectively referred to as being space-time stationary). The homogeneity and stationarity assumptions imply that $\boldsymbol{c}\left(\mathbf{x}, t ; \mathbf{x}^{\prime}, t^{\prime}\right)$ depends only on the difference between the space and time arguments, i.e., the spatial separation vector $\boldsymbol{\rho}=\mathbf{x}-\mathbf{x}^{\prime}$ and the lag $\tau=t-t^{\prime}$. Isotropy further restricts the dependence to the form

$$
c\left(\mathbf{x}, t ; \mathbf{x}^{\prime}, t^{\prime}\right)=c(\rho, \tau),
$$

where $\rho=|\boldsymbol{\rho}|$. We should note that in the present paper the stationarity property refers only to the second moment statistics rather than the full underlying probability distribution. The spatial Fourier amplitudes

$$
a(\mathbf{k}, t)=(2 \pi)^{-1} \int \mathrm{d}^{2} \mathbf{x} e^{-i \mathbf{k} \cdot \mathbf{x}} R^{\prime}(\mathbf{x}, t)
$$

are, in general, complex but constrained to satisfy the condition $a^{*}(\mathbf{k}, t)=a(-\mathbf{k}, t) \quad$ (where asterisk denotes complex conjugation), which follows from the fact that $R^{\prime}(\mathbf{x}, t)$ is real. We assume that the $a(\mathbf{k}, t)$ evolve in time according to the generalized Langevin equation

$$
{ }_{-\infty} D_{t}^{\beta} a(\mathbf{k}, t)=-\tau_{k}^{-\beta} a(\mathbf{k}, t)+f(\mathbf{k}, t) .
$$

Here ${ }_{-\infty} D_{t}^{\beta}$ denotes the Liouville-Weyl fractional derivative operator of order $\beta$ with respect to the argument $t$, which can be regarded as a shorthand for the operator defined in equation (A2) with the lower limit of integration tending to $-\infty$. See Appendix A for a brief account of some basic results from the calculus of fractional derivatives. From the definition, it is clear that equation (4) is actually an integro-differential equation and therefore represents nonlocal time evolution. Also, in equation (4), $f(\mathbf{k}, t)$ represents a white-noise forcing term with zero mean and $\delta$-function covariance

$$
\left\langle f(\mathbf{k}, t) f^{*}\left(\mathbf{k}^{\prime}, t^{\prime}\right)\right\rangle=(2 \pi)^{3 / 2} F_{0} \delta\left(\mathbf{k}-\mathbf{k}^{\prime}\right) \delta(\tau),
$$

and

$$
\tau_{k}=\tau_{0}\left(1+k^{2} L_{0}^{2}\right)^{-\alpha / 2}
$$

is the relaxation time for the Fourier mode $\mathbf{k}$ depending only on the wave number $k=|\mathbf{k}|$ by virtue of spatial isotropy. [Note the incorrect normalization in KB03 equation (5).] In the frequency domain, equation (5) is equivalent to

$$
\left\langle f(\mathbf{k}, \omega) f^{*}\left(\mathbf{k}^{\prime}, \omega^{\prime}\right)\right\rangle=(2 \pi)^{3 / 2} F_{0} \delta\left(\mathbf{k}-\mathbf{k}^{\prime}\right) \delta\left(\omega-\omega^{\prime}\right),
$$

$f(\mathbf{k}, \omega)$ being the temporal Fourier transform of $f(\mathbf{k}, t)$. (We will occasionally denote a function and its Fourier transform by the same symbol when there is no possibility of confusion). Here $F_{0}$ is a strength parameter, and $\tau_{0}$ and $L_{0}$ are characteristic time and length scale parameters, respectively. The characteristic length scale $L_{0}$ effectively separates the rain field fluctuations into two regimes: a short wavelength (large $k$ ) scaling regime in which $\tau_{k}$ tends to zero according to a power law $k^{-\alpha}$ and a long wavelength (small $k$ ) regime in which $\tau_{k}$ approaches $\tau_{0}$. Physically, $\tau_{0}$ represents the duration of an average rain event. Equations (4)-(6) are the basic equations of the model. The three quantities $F_{0}, \tau_{0}$, and $L_{0}$ together with the two dimensionless exponents $\alpha$ and $\beta$ define the full set of model parameters. Selection of the lower limit of time integration as $-\infty$ is dictated by the condition that we want the model to describe stationary temporal statistics, where there is no preferred choice of the initial time. Choosing any finite value would correspond to choosing a particular time at which the initial condition for the stochastic equation has to be set, thus leading to loss of stationarity. The rain rate field $R^{\prime}(\mathbf{x}, t)$ itself defined by the inverse spatial Fourier transform of (3) satisfies a stochastic field equation involving fractional spatial and temporal derivative operators. The spatial derivative operator that results from a spatial Fourier transform of equation (4) with $\tau_{k}$ given by equation (6) can be formally represented as the familiar Helmholtz operator $\left(-\nabla^{2}+L_{0}^{-2}\right)$ raised to the power $\alpha \beta / 2$.

[14] The "old" spectral model of BK96 and KB03 is recovered in the special case $\beta=1$, in which the derivative operator ${ }_{-\infty} D_{t}^{\beta=1}$ reduces to the ordinary time derivative $\mathrm{d} / \mathrm{d} t$. Equation (3) then simply becomes an ordinary first-order stochastic differential equation with exponential relaxation. For a general noninteger order, the power law kernel of the fractional derivative operator is indicative of an underlying random process that is non-Markovian. The process is now characterized by a nonexponential relaxation. The response by the 
precipitation process to a unit impulse is determined by the Green's function $G\left(\mathbf{k}, t-t^{\prime}\right)$, which is the solution to the inhomogeneous equation

$$
\left({ }_{-\infty} D_{t}^{\beta}+\tau_{k}^{-\beta}\right) G\left(\mathbf{k}, t-t^{\prime}\right)=\delta\left(t-t^{\prime}\right)
$$

[15] The Fourier amplitudes $a(\mathbf{k}, t)$ have zero mean and lagged covariance of the form

$$
\left\langle a(\mathbf{k}, t) a^{*}\left(\mathbf{k}^{\prime}, t^{\prime}\right)\right\rangle=2 \pi c(k, \tau) \delta\left(\mathbf{k}-\mathbf{k}^{\prime}\right),
$$

where, as a consequence of spatial isotropy, we can write $c(k, \tau)=c(\mathbf{k}, \tau)$ for the spatial Fourier transform of $c(\rho, \tau)$. In the frequency domain, equation (9) corresponds to

$$
\left\langle a(\mathbf{k}, \omega) a^{*}\left(\mathbf{k}^{\prime}, \omega^{\prime}\right)\right\rangle=(2 \pi)^{3 / 2} S(k, \omega) \delta\left(\mathbf{k}-\mathbf{k}^{\prime}\right) \delta\left(\omega-\omega^{\prime}\right),
$$

where we have introduced the power spectrum of the rain field fluctuations $S(k, \omega)$ as the temporal Fourier transform of $c(k, \tau)$. From the correspondence ${ }_{-\infty} D_{t}^{\beta} \Leftrightarrow(-i \omega)^{\beta}$ under the action of the Fourier transform (see Appendix A), it follows that

$$
S(k, \omega)=\frac{F_{0}}{\left|(-i \omega)^{\beta}+\tau_{k}^{-\beta}\right|^{2}},
$$

which can be simplified to the form

$$
S(k, \omega)=F_{0}\left[|\omega|^{2 \beta}+2 \cos (\beta \pi / 2)|\omega|^{\beta} \tau_{k}^{-\beta}+\tau_{k}^{-2 \beta}\right]^{-1} .
$$

The principal branch of the multivalued function in the denominator is assumed to be in the range $-\pi<\operatorname{Arg} \omega \leq \pi$.

[16] The spectrum $S(k, \omega)$ given by equation (11) yields the full set of second moment statistics of the rain rate field. The point covariance function $c(\rho, \tau)$ is the space-time Fourier transform of the spectrum, i.e.,

$$
c(\rho, \tau)=(2 \pi)^{-3 / 2} \int_{-\infty}^{\infty} \mathrm{d} \omega \int_{\mathrm{d}^{2}} \mathbf{k} e^{i(\mathbf{k} \cdot \boldsymbol{\rho}-\omega \tau)} S(k, \omega) .
$$

It can be evaluated in two steps. First, consider the temporal Fourier transform of $S(k, \omega)$ :

$$
c(k, \tau)=(2 \pi)^{-1 / 2} \int_{-\infty}^{\infty} \mathrm{d} \omega e^{-i \omega \tau} S(k, \omega) .
$$

From the explicit form (11) of the spectrum, it follows, by a simple scaling argument, that $c(k, \tau)$ has the functional form

$$
c(k, \tau)=g(\beta) F_{0} \tau_{k}^{2 \beta-1} h\left(|\tau| / \tau_{k}\right)
$$

where $h(x)$ is a certain transcendental function defined in Appendix $\mathrm{B}$ and $g(\beta)$ is a normalization factor adjusted so that $h(0)=1$. When $\beta=1, h(x)$ is simply $\exp (-x)$. Unfortunately in the $\beta \neq 1$ case, it does not appear possible to express $h(x)$ in terms of familiar analytical functions. The forms of the function $h(x)$ for some typical values of $\beta$ obtained by numerical integration are shown in Appendix B (see Figure B1).
[17] The space-time covariance $c(\rho, \tau)$ is given by the spatial Fourier transform of $c(k, \tau)$. Spatial isotropy allows one to carry out the angular integration in the k-plane. This yields

$$
c(\rho, \tau)=\int_{0}^{\infty} \mathrm{d} k k J_{0}(k \rho) c(k, \tau),
$$

where $J_{0}(x)$ is the usual Bessel function of order zero. The analytical form (13) implies that the space-time covariance function $c(\rho, \tau)$ given by (14) is in general not factorizable into spatial and temporal dependence. Upon setting $\tau=0$, we obtain the spatial covariance function $c(\rho, 0)$ in the form of an integral that is identical with the one encountered in the $\beta=1$ case. It can be similarly evaluated with the result

$$
c(\rho, 0)=\gamma_{0} C_{v}\left(\rho / L_{0}\right)
$$

provided we now identify the index $v$ through the formula

$$
\alpha(2 \beta-1)=2(1+v)
$$

where, as in BK96 and KB03, we introduce

$$
C_{v}(z)=(z / 2)^{v} K_{v}(z)
$$

$K_{v}(z)$ being the modified Bessel function of order $v$. For a purely spatial random process, a class of covariance functions of the form (15) was originally introduced by Materrn $[1960,1986]$. The factor $\gamma_{0}$ is now a slightly more complicated quantity with the dimension of [rain rate $]^{2}$ that is expressible in terms of the basic model parameters:

$$
\gamma_{0}=\frac{g(\beta) F_{0} \tau_{0}^{2 \beta-1}}{L_{0}^{2} \Gamma(1+v)},
$$

where $\Gamma(z)$ denotes the Euler gamma function. The temporal covariance of the rain rate field can be obtained by simply setting $\rho=0$ in equation (14):

$$
c(0, \tau)=\int_{0}^{\infty} \mathrm{d} k k c(k, \tau) .
$$

[18] The point variance $c(0,0) \equiv \sigma_{0}^{2}$ is evaluated by letting $\rho \rightarrow 0, \tau \rightarrow 0$ in equation (14) and making use of equation (13). In terms of the dimensionless variable $y=1+k^{2} L_{0}^{2}$, it takes the form

$$
\sigma_{0}^{2}=(1 / 2) \gamma_{0} \Gamma(1+v) \int_{1}^{\infty} \mathrm{d} y y^{-(1+v)} .
$$

The integral converges when $v>0$, i.e., when $\alpha(2 \beta-1)>2$ yielding $\sigma_{0}^{2}=\gamma_{0} \Gamma(v) / 2$, but diverges when $v \leq 0$ causing $\sigma_{0}^{2}$ to be infinite. As was already discussed in BK96 and KB03, when $v<0$, the spatial covariance $c(\rho, 0)$ has a power law singularity at $\rho=0: c(\rho, 0) \sim\left(\rho / L_{0}\right)^{-2|v|}$, which weakens to logarithmic when $v=0$ [North and Nakamoto, 1989]. Later in section 3, as we examine the statistical properties of precipitation data sets, it will become clear that the model fit to data strongly favors the $v<0$ case.

[19] While the point rain statistics themselves cannot be directly measured, they determine the covariance statistics of the space- or time-averaged rain rate data that can be measured. The next two subsections summarize their properties. 


\subsection{Covariance Statistics of Area-Averaged Rain Rate}

[20] Now we consider the space-time covariance of spatially averaged radar rainfall data. A gridded radar precipitation data set consists of a sequence of images, each image being an array of $L \times L$ square grid boxes in which the average (near-instantaneous) rain rate is specified. Let

$$
r_{A}(t)=\left(1 / L^{2}\right) \int_{A} \mathrm{~d}^{2} \mathbf{x} R(\mathbf{x}, t)
$$

denote the average rain rate in an $L \times L$ square $A$ at time $t$. The space-time covariance between the rain rates in two squares $A$ and $A^{\prime}$ of equal area $L^{2}$, whose centers are separated by a distance vector $\mathbf{s}$ at two different times $t$ and $t+\tau$, is defined as

$$
\Gamma_{A A^{\prime}}(\mathbf{s}, \tau)=\left\langle r_{A}^{\prime}(t) r_{A^{\prime}}^{\prime}(t+\tau)\right\rangle
$$

where, as before, the prime on the rain rate variables indicate deviation from the mean. It can be written as a double areaintegral over the space-time covariance function of the point rain rate:

$$
\Gamma_{A A^{\prime}}(\mathbf{s}, \tau)=\left(1 / L^{4}\right) \int_{A} \mathrm{~d}^{2} \mathbf{x} \int_{A^{\prime}} \mathrm{d}^{2} \mathbf{x}^{\prime} c\left(\mathbf{s}+\mathbf{x}^{\prime}-\mathbf{x}, \tau\right) .
$$

Evaluating equation (22) for $\mathbf{s}=0, \tau=0$, one obtains the variance of area-averaged rain rate $\sigma_{A}^{2} \equiv\left\langle r_{A}^{\prime 2}\right\rangle$. The spatial correlation between two boxes $A$ and $A^{\prime}$ separated by $\mathbf{s}$ is then given by $\Phi_{A A^{\prime}}(\mathbf{s})=\Gamma_{A A^{\prime}}(\mathbf{s}, 0) / \sigma_{A}^{2}$.

[21] The time dependence of the lagged autocorrelation function $\Phi_{A A}(\tau)=\Gamma_{A A}(0, \tau) / \sigma_{A}^{2}$ defines a characteristic timescale, the integral correlation time for area-averaged rain rate

$$
\tau_{A}=\int_{0}^{\infty} \mathrm{d} \tau \Phi_{A A}(\tau) .
$$

For an exponentially decaying correlation, $\tau_{A}$ is simply the (1/e)-folding time. However, our model-predicted correlation functions are markedly nonexponential.

[22] Explicit formulas for the various second moment statistics including $\sigma_{A}^{2}$ and $\tau_{A}$ are given in Appendix B.

\subsection{Covariance Statistics of Time-Averaged Rain Rate}

[23] Next we examine how the model represents the statistics of rain gauge data. While the actual quantity measured depends on the specific type of instrument, the data is usually converted into a form that can be idealized as the time-averaged rain rate at a point, i.e.,

$$
r_{T}(\mathbf{x})=(1 / T) \int_{0}^{T} \mathrm{~d} t R(\mathbf{x}, t) .
$$

In general, one could consider the space-time covariance $\Gamma_{T T^{\prime}}$ $(\rho, \tau)$ between rain rates at two points separated by a distance $\rho$, time-averaged over two intervals $T$ and $T^{\prime}$ whose midpoints are separated by a lag $\tau$ (see Appendix B). For convenience we restrict ourselves to the zero-lag case, namely the spatial covariance of the rain rate averaged over a time interval $[0, T]$ at two points with separation $\rho$

$$
\begin{aligned}
\Gamma_{T T}(\rho, 0) & =\left\langle r_{T}^{\prime}(\mathbf{x}) r_{T}^{\prime}\left(\mathbf{x}^{\prime}\right)\right\rangle \\
& =\left(1 / T^{2}\right) \int_{0}^{T} \mathrm{~d} t \int_{0}^{T} \mathrm{~d} t^{\prime} c\left(\rho, t-t^{\prime}\right),
\end{aligned}
$$

which can be reduced to a single integral

$$
\Gamma_{T T}(\rho, 0)=(2 / T) \int_{0}^{T} \mathrm{~d} \tau(1-\tau / T) c(\rho, \tau) .
$$

The limit $\rho \rightarrow 0$ yields the variance of time-averaged rain rate

$$
\sigma_{T}^{2} \equiv\left\langle r_{T}^{\prime 2}\right\rangle=(2 / T) \int_{0}^{T} \mathrm{~d} \tau(1-\tau / T) c(0, \tau) .
$$

[24] From equations (25) and (26) we can compute the spatial correlation function of gauge pairs as a function of separation: $\Psi_{T T}(\rho)=\Gamma_{T T}(\rho, 0) / \sigma_{T}^{2}$. For explicit evaluation of these quantities, it is again convenient to go over to the Fourier representation. The resulting formulas are given in Appendix B.

\subsection{The Power Law Scaling Regime in the Case $v<0$}

[25] As already noted, the space-time covariance $c(\rho, \tau)$ predicted by the model becomes singular in the limit of small $\rho$ and $\tau$ when $v \leq 0$ but approaches a well-defined finite value when $v>0$. In the case $v<0$, which is of greater interest to us, this singular behavior at small space-time separations $\rho$, $\tau$ indicates the presence of a "scaling regime" in which the model exhibits approximate scale-invariance under a spacetime scaling $\rho \rightarrow \lambda \rho, \tau \rightarrow \lambda^{\alpha} \tau$. This is elucidated by examining the limiting form of the spectrum $S(k, \omega)$ in the limit of large $k$ and $\omega$, which we denote by $S^{(\infty)}(k, \omega)$. We find that $S^{(\infty)}(k, \omega)$ is invariant (up to an overall multiplicative factor) under a scale transformation in the Fourier space $k \rightarrow \lambda^{-1} k, \omega \rightarrow \lambda^{-\alpha} \omega$ :

$$
S^{(\infty)}\left(\lambda^{-1} k, \lambda^{-\alpha} \omega\right)=\lambda^{2 \alpha \beta} S^{(\infty)}(k, \omega) .
$$

Its Fourier transform $c^{(\infty)}(\rho, \tau)$, which is the asymptotic form of the exact space-time covariance $c(\rho, \tau)$, satisfies

$$
c^{(\infty)}\left(\lambda \rho, \lambda^{\alpha} \tau\right)=\lambda^{-2|v|} c^{(\infty)}(\rho, \tau) .
$$

As the scale factor $\lambda \rightarrow 0$, one attains larger and larger values of $k$ and $\omega$ corresponding to smaller and smaller space-time scales. Choosing the scale factor to be $\lambda=1 / \rho_{*}$, we immediately conclude from equation (28) that $c^{(\infty)}(\rho, \tau)$ must have the functional form

$$
c^{(\infty)}(\rho, \tau)=\gamma_{0} \rho_{*}{ }^{-2|v|} \varphi\left(\tau_{*} / \rho_{*}^{\alpha} ; \alpha, \beta\right),
$$

where, for convenience, we have introduced the dimensionless variables $\rho_{*}=\rho / L_{0}, \tau_{*}=\tau / \tau_{0}$. The type of combined space-time scaling property expressed by equation (28) is frequently referred to as dynamic scaling, $\alpha$ being the corresponding scaling exponent. The scaling function $\varphi(\xi ; \alpha, \beta)$ also depends explicitly on both $\alpha$ and $\beta$. The limiting behavior of $c^{(\infty)}(\rho, \tau)$ as $\rho_{*}, \tau_{*} \rightarrow 0$ is nonuniform, i.e., dependent on the direction from which the space-time origin is approached. The double limit is characterized by the functional dependence of $\varphi(\xi ; \alpha, \beta)$ on the scaling variable $\xi=\tau_{*} / \rho_{*}^{\alpha}$. In particular, the behavior of $c^{(\infty)}(\rho, \tau=0)$ as $\rho_{*} \rightarrow 0$ is determined by the asymptotic behavior of $\varphi(\xi ; \alpha, \beta)$ near $\xi=0$. Similarly, the behavior of $c^{(\infty)}(\rho=0, \tau)$ as $\tau * \rightarrow 0$ is determined by the asymptotic behavior of $\varphi(\xi ; \alpha, \beta)$ as $\xi \rightarrow \infty$. From the exact result for the spatial covariance $c(\rho, \tau=0)=\gamma_{0} C_{v}(\rho *)$ [see equation (15)], making use of 


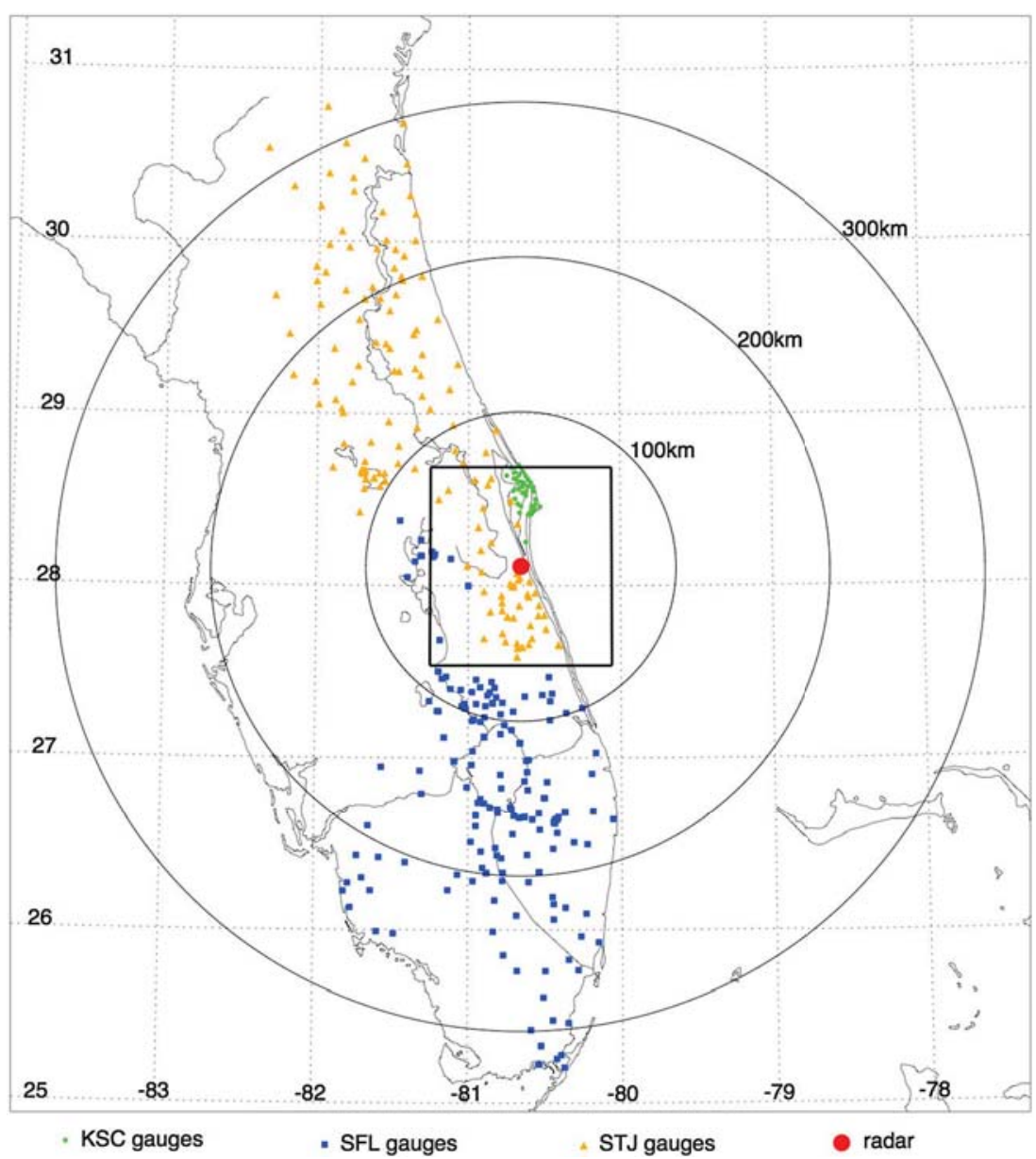

Figure 1. Map showing the locations of the MELB radar and the tipping bucket rain gauges in Florida operated as part of the TRMM ground validation program. Also shown are the radial distance contours of the radar FOV and the position of the $128 \mathrm{~km}$ area from which statistics is collected.

the asymptotic behavior of $C_{v}(z)$ as $z \rightarrow 0$ when $v<0$, we get the limit

$$
\varphi(0 ; \alpha, \beta)=2^{-(1+2|v|)} \Gamma(|v|)
$$

[26] Unlike the $\beta=1$ case studied in BK96 and KB03, $c(\rho=0, \tau)$ likely cannot in general be expressed in closed form. However, scaling arguments like the one above can also be invoked to obtain the asymptotic $\tau$-dependence of $c^{(\infty)}(\rho=0, \tau)$ as $\tau \rightarrow 0$. Setting $\rho=0$ and choosing the scale factor $\lambda=\tau_{*}^{-1 / \alpha}$ in equation (28), we get the asymptotic form

$$
c^{(\infty)}(\rho=0, \tau) \sim \gamma_{0} K \tau_{*}^{-2|v| / \alpha}
$$

as $\tau_{*} \rightarrow 0$, where $K$ is a dimensionless constant. This is compatible with the general form of equation (29) if and only if $\varphi(\xi ; \alpha, \beta)$ has the falloff

$$
\varphi(\xi ; \alpha, \beta) \underset{\xi \rightarrow \infty}{\longrightarrow} K \xi^{-2|v| / \alpha}
$$

The asymptotic behavior of the area and time-averaged statistics can be deduced by replacing the exact space-time correlation $c(\rho, \tau)$ by its scaling approximation $c^{(\infty)}(\rho, \tau)$ in the area/time integrals. The details are relegated to Appendix B.

[27] The scaling properties of the space-time covariance in the $\beta=1$ case were explored in greater detail by Kundu and Bell [2006].

\subsection{Effect of a Short Distance Cut-Off}

[28] As will be found later, a problem arises when we examine the spectral model predictions in light of the data. We find that the growth property of the space-time covariance at small space-time scales in the $v<0$ case predicted by the spectral model is broadly consistent with data, but only up to a certain point. The spatial variance $\sigma_{A}^{2}$ estimated from radar data does appear to follow the predicted power law behavior [see equation (B22)] within the accessible range of spatial scales. But rain gauge data, which allows one to access much smaller space-time scales, exhibit a more complicated behavior. The scale dependence of the temporal variance $\sigma_{T}^{2}$ follows the model prediction up to a certain minimum averaging time $T$ of about $30 \mathrm{~min}$, but increasingly deviates from it at shorter time scales. As $T \rightarrow 0$, instead of growing in accordance with the predicted power law growth, $\sigma_{T}^{2}$ appears to gradually approach a finite asymptotic value $\sigma_{0}^{2}$, the point variance, indicating a steeper decrease of the 
Table 1. Model Parameters and Related Quantities for the Spectral Model

\begin{tabular}{|c|c|c|c|c|c|c|c|c|c|c|}
\hline Radar & Season & $(R)\left(\mathrm{mm} \mathrm{h}^{-1}\right)$ & $\alpha$ & $\beta$ & $v$ & $\gamma_{0}\left(\mathrm{~mm}^{2} \mathrm{~h}^{-2}\right)$ & $L_{0}(\mathrm{~km})$ & $\tau_{0}(\min )$ & $\sigma_{0}^{2}\left(\mathrm{~mm}^{2} \mathrm{~h}^{-2}\right)$ & $\Lambda(\mathrm{km})$ \\
\hline KWAJ & MAM 2001 & 0.098 & 0.99 & 1.18 & -0.327 & 0.019 & 281 & 775 & 2.5 & 0.48 \\
\hline KWAJ & JJA 2001 & 0.232 & 0.93 & 1.28 & -0.279 & 0.060 & 438 & 770 & 7 & 0.36 \\
\hline KWAJ & SON 2001 & 0.357 & 0.99 & 1.24 & -0.265 & 0.213 & 136 & 381 & 13 & 0.27 \\
\hline KWAJ & DJF 2002 & 0.114 & 1.40 & 1.00 & -0.298 & 0.067 & 72.1 & 524 & 3.5 & 0.32 \\
\hline MELB & DJF 2001 & 0.028 & 1.17 & 1.18 & -0.202 & 0.030 & 69.0 & 196 & 1.2 & 0.07 \\
\hline MELB & MAM 2001 & 0.104 & 1.17 & 1.26 & -0.113 & 0.348 & 73.2 & 197 & 6 & 0.09 \\
\hline MELB & JJA 2001 & 0.214 & 1.14 & 1.26 & -0.130 & 1.078 & 33.9 & 98.8 & 13 & 0.19 \\
\hline MELB & SON 2001 & 0.183 & 1.12 & 1.20 & -0.218 & 0.337 & 51.5 & 209 & 10 & 0.18 \\
\hline
\end{tabular}

characteristic time scale $\tau_{k}$ than the model-predicted $k^{-\alpha}$ falloff at large $k$. On the other hand, the $v>0$ case of the model, which would have accounted for this effect, does not generally fit the radar statistics. A simple way to incorporate the desired modification at short length and time scales is to assume that instead of equation (6), $\tau_{k}$ is given by the relation

$$
\tau_{k}= \begin{cases}\tau_{0}\left(1+k^{2} L_{0}^{2}\right)^{-\alpha / 2} & ; k<1 / \Lambda \\ 0 & ; k>1 / \Lambda\end{cases}
$$

where $\Lambda$ is a short distance ("ultraviolet") cut-off. Physically this means that the spatial Fourier modes of the precipitation field $a(\mathbf{k}, t)$ of wavelength shorter than $2 \pi \Lambda$ are damped out instantly. Accordingly, the spectrum $S(k, \omega)$ and its temporal Fourier transform $c(k, \tau)$ also vanish for wave numbers $k>1 / \Lambda$. In a more realistic model that describes the smallscale behavior of rain, the sharp cut-off introduced in equation (33) may have to be appropriately modified based on empirical evidence. The possibility of a steep decrease of the spectrum beyond the scaling regime is reminiscent of a similar phenomenon in fluid turbulence, namely breakdown of the famous Kolmogorov scaling of the energy spectrum beyond the inertial range [Frisch, 1995]. In order to obtain an estimate of $\Lambda$, we return to equation (19) for the point variance. Explicit evaluation of the integral with equation (33) leads to the simple analytic result:

$$
\sigma_{0}^{2}=\frac{1}{2} \gamma_{0}|\Gamma(v)| \cdot\left[\left(1+L_{0}^{2} / \Lambda^{2}\right)^{|v|}-1\right] .
$$

The spatial cut-off $\Lambda$ is easily obtained from equation (34) if a reasonable value of $\sigma_{0}^{2}$ can be estimated by extrapolation from the gauge data.

\section{Comparison Between the Model and Observations}

\subsection{Radar Data Analysis}

[29] The parameters of the model can be conveniently estimated from a gridded radar precipitation data set. We have fit the model to the data sets (TRMM standard product 2A-53) constructed from radar images collected from the KWAJ and MELB radars located respectively at $\left(8.718^{\circ} \mathrm{N}, 167.733^{\circ} \mathrm{E}\right)$ and $\left(28.113^{\circ} \mathrm{N}, 80.654^{\circ} \mathrm{W}\right.$ ) (Figure 1). Next we summarize some relevant features of these data sets.

[30] For both the KWAJ and MELB radars, the FOV consists of a circular area of diameter $300 \mathrm{~km}$. The data was gridded at a $2 \mathrm{~km} \times 2 \mathrm{~km}$ spatial resolution. In order to reduce

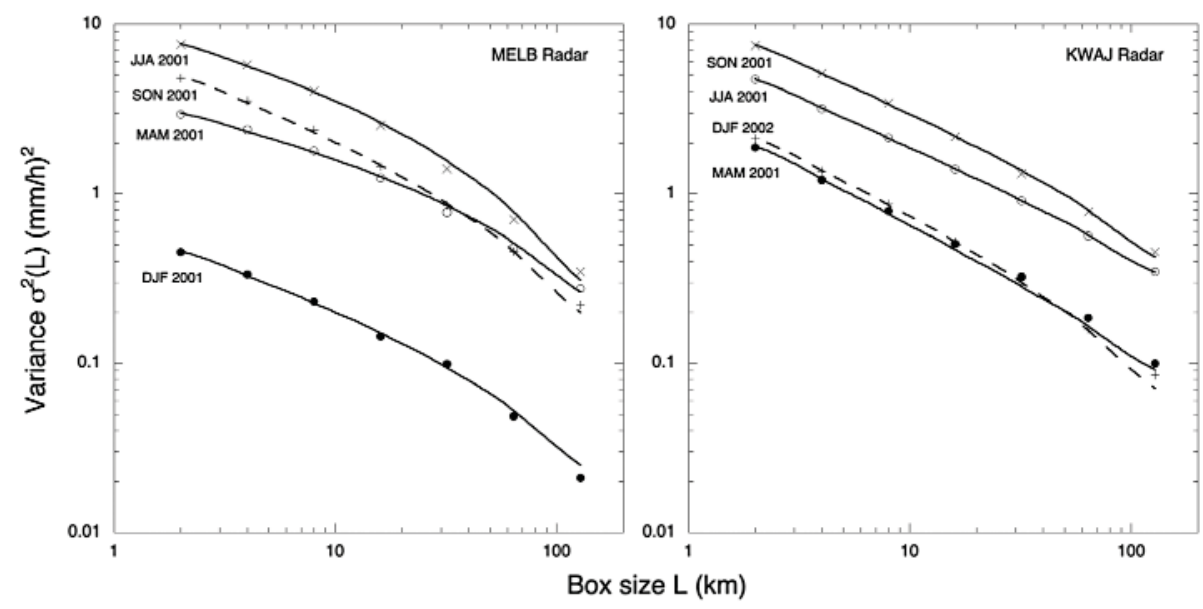

Figure 2. Plot of the variance of area average rain rate $\sigma_{\mathrm{A}}^{2} \equiv \sigma^{2}(L)$ as a function of the spatial averaging scale $L$ estimated from (left) the Melbourne radar data for the four seasons Winter (December 2000-February 2001), Spring (March-May 2001), Summer (June-August 2001), and Autumn (September-November 2001) and from (right) the KWAJ radar for the four seasons Spring (March-May 2001), Summer (June-August 2001) and Autumn (September-November 2001), and Winter (December 2001-February 2002) superimposed on the curves predicted from the model formula (B7) with the parameters listed in Table 1. 


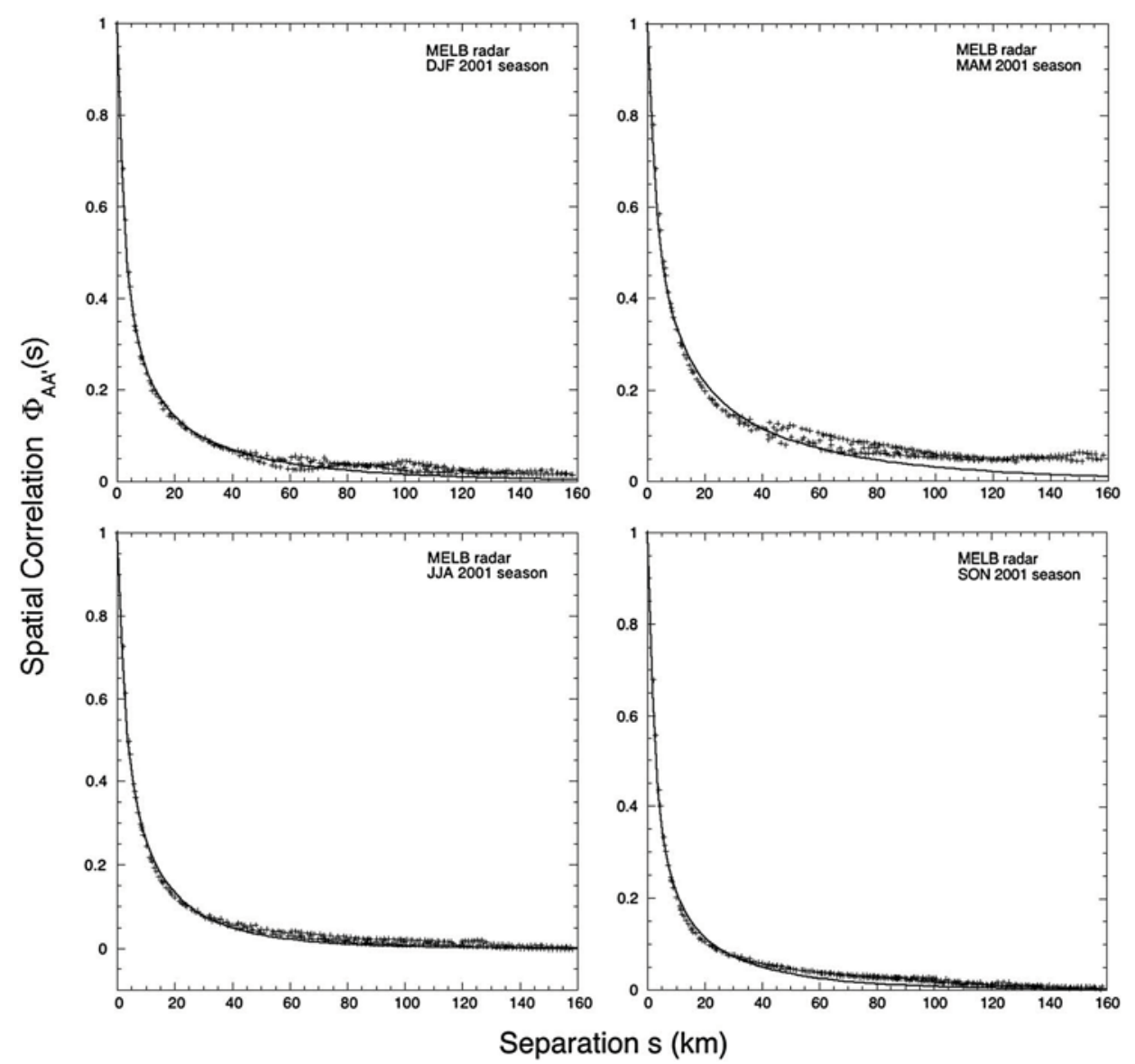

Figure 3. Comparison of the spatial correlations of $2 \mathrm{~km}$ radar pixels as a function of the separation $s$ as estimated from the Melbourne radar data (the + symbols) and the spectral model (the solid curve) with the parameters listed in Table 1 for four seasons spanning the period December 2000 to November 2001 as in Figure 2.

the uncertainties in rain retrieval due to radar attenuation with distance, statistics were computed from a $128 \mathrm{~km} \times 128 \mathrm{~km}$ area centered at the radar location by aggregating the data at spatial scales $L=2,4,8, \ldots, 128 \mathrm{~km}$. Only those grid boxes within the selected area that had at least $95 \%$ valid pixels were included in the sample. This helped eliminate boxes at the smaller scales 4,8 , and $16 \mathrm{~km}$ located near the center, which occasionally suffered from data dropout because of ground clutter.

[31] The temporal sampling pattern of the radar has an important effect on the estimation of rain statistics. The MELB radar scans were carried out at different speeds during the quiet and the active periods of rainfall. Rainfall images were available from it every $5 \mathrm{~min}$ during the active periods and every 10 min during the quiet periods. Images from the KWAJ radar, which was operated at a single speed, are spaced in a somewhat more irregular manner: the temporal spacing between the consecutive images predominantly alternated between 5 and $7 \mathrm{~min}$ long gaps.

[32] The various space-time statistics of the TRMM 2A-53 data needed for the analysis (the variance and covariance functions) were computed using routines provided in the $\mathrm{R}$ statistical software package. For the spatial analysis we computed the variances $\sigma_{A}^{2}$ for various box sizes $L$ between 2 and $128 \mathrm{~km}$ and the spatial autocorrelation function $\Phi_{A A^{\prime}}(\mathbf{s})$ for pairs of $L=2 \mathrm{~km}$ radar pixels. For simplicity the measured spatial correlation function is regarded as a function of the separation $s=|\mathbf{s}|$ in accordance with our spatial isotropy assumption. Since all the available pairs within the $128 \mathrm{~km}$ area are included in the computation of $\Phi_{A A^{\prime}}(\mathbf{s})$, our data analysis automatically averages over all pair locations within the box and allows us to assume spatial homogeneity. The direction dependence was implicitly averaged over two mutually perpendicular directions in this step by pooling together all pairs with the same separation $s$.

[33] One effect of the uneven temporal sampling of different portions of a data set like that of the MELB radar is that it introduces a systematic bias into the usual estimates of many statistics. In particular, the "simple" estimates of the elementary statistics at a spatial scale $L$, such as the unconditional mean $\langle R\rangle$ and the probability of nonzero rain $p(L)=\operatorname{Pr}\left[r_{A}>0\right]$ obtained by summing the corresponding data and dividing by the actual number of samples $N(L)$, are biased high. One can obtain "improved" estimates of these statistics that reduce this bias as follows. We assume that the temporal spacing between two successive images $\Delta t_{2}$ during a quiet period (labeled 2), which is mostly dry, is larger than the spacing $\Delta \mathrm{t}_{1}$ during an active period (labeled 1) when rain occurs more frequently. Suppose $\Delta t_{2}=w \Delta t_{1}$; in the case of the MELB radar, $w=2$. For each stretch of the time series of radar images 


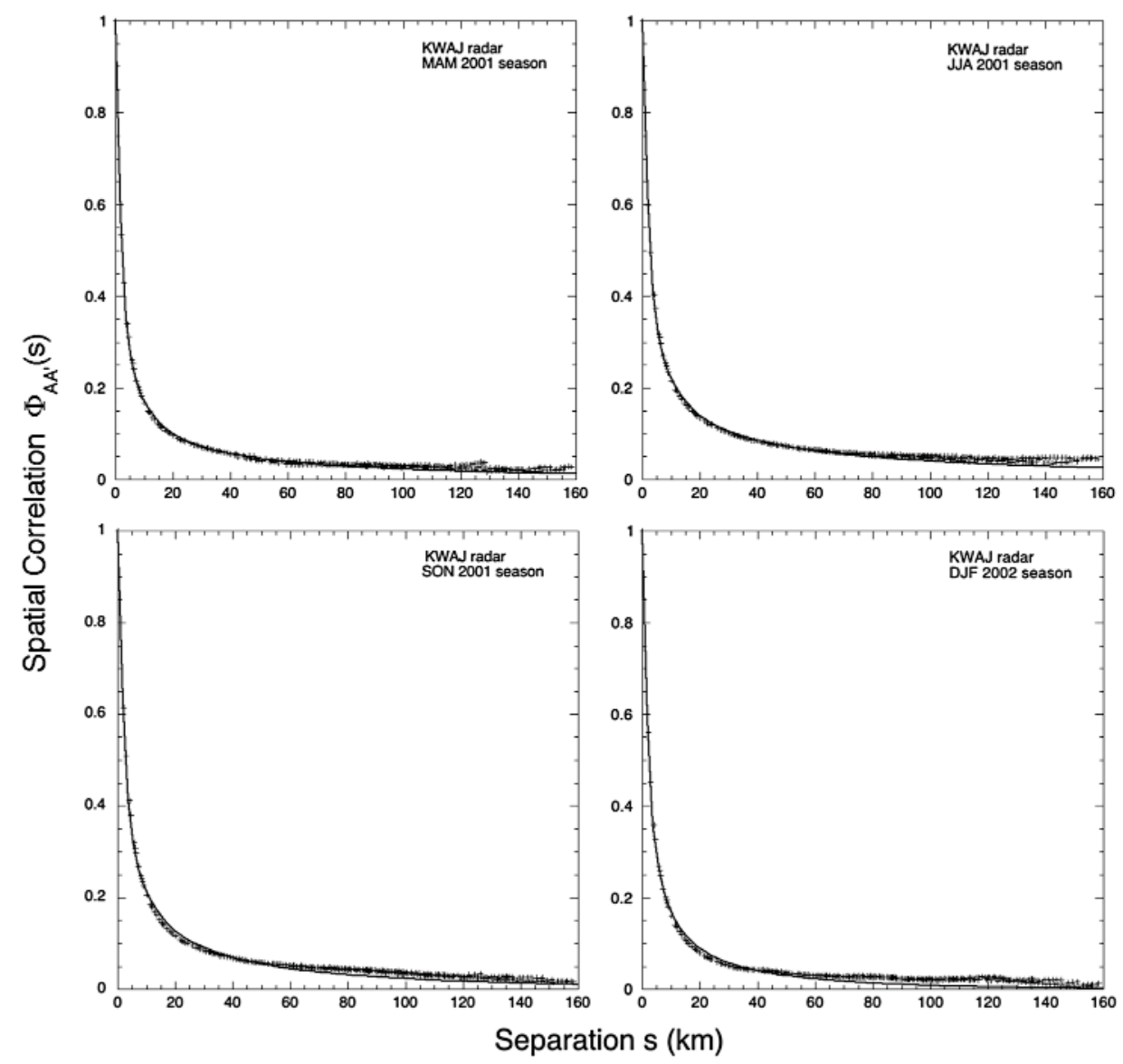

Figure 4. Same as in Figure 3 but for the KWAJ radar data (the + symbols) and the spectral model (the solid curve) for the four seasons spanning the period March 2001 to February 2002.

during a quiet period, we augment it by padding each gap successively with a segment consisting of $(w-1)$ additional copies of the initial point spaced so that the new series has a constant time step $\Delta t_{1}$.

[34] We can get a rough estimate of the bias from uneven sampling in the following way. Let us write $N=N_{1}+N_{2}$, where $N_{1}$ and $N_{2}$ are the actual number of samples during the active and the quiet periods, respectively. For the nonzero rain probability $p$, instead of the naive estimate

$$
p=\frac{N_{1}^{r>0}+N_{2}^{r>0}}{N_{1}+N_{2}},
$$

we now have the adjusted estimate

$$
p^{\prime}=\frac{N_{1}^{r>0}+w N_{2}^{r>0}}{N_{1}+w N_{2}}=\left[N p+(w-1) N_{2}^{r>0}\right] / N^{\prime},
$$

where $N_{1}^{r>0}$ and $N_{2}^{r>0}$ are the number of rainy samples during the active and quiet periods, respectively, and $N^{\prime}=N_{1}+w N_{2}$ is the total number of samples in the augmented series. If the number of rainy samples occurring during the quiet period is small, i.e., $N_{2}^{r>0} \ll N^{\prime}$, then we get the approximate result $p^{\prime} \approx\left(N / N^{\prime}\right) p$. The same correction factor $\left(N / N^{\prime}\right)$ approximately applies to the unconditional mean $\langle R\rangle$ and the various unconditional moments $M_{q}(L) \equiv\left\langle r_{L}^{q}\right\rangle$ under the similar condition that the contribution from rain occurring during the quiet periods can be neglected.
[35] The lagged autocorrelation $\Phi_{A A}(\tau)$ was computed for several averaging scales $L$ between 2 and $128 \mathrm{~km}$ and for various lags $\tau$ from the sequence of radar images. As expected, it was found that observed autocorrelation in general exhibits fluctuation due to sampling uncertainty, which becomes large especially at those $\tau$ values where one has relatively few samples. For our purpose, however, only those lags for which the sampling error is small enough to be negligible were considered. In order to reduce the effect of the sampling error, we had to restrict ourselves to lags for which the number of available samples exceeds a judiciously chosen value. The estimates at the smaller scales $L=2,4,8, \ldots, 64 \mathrm{~km}$ were obtained by partitioning the $128 \mathrm{~km}$ area into nonoverlapping subareas of size $L \times L$ and averaging over the computed lagged autocorrelation for each subarea. Pooling together samples of area-pairs for a fixed lag $\tau$ for the temporal autocovariance also presumes temporal stationarity of the statistics.

\subsection{Fitting the Model to Radar Rainfall Statistics}

[36] Estimation of the model parameters presented a difficult problem even in the simpler $\beta=1$ case considered in BK96. Not surprisingly, the general $\beta$ case proves to be even more challenging. Since the parameters appear directly in the spectrum, one's first thought might be to perform a spectral analysis of the pixel data and estimate the spectrum of the point rain rate from it. One could then fit the observed spectrum to equation (11) and estimate the model parameters. 

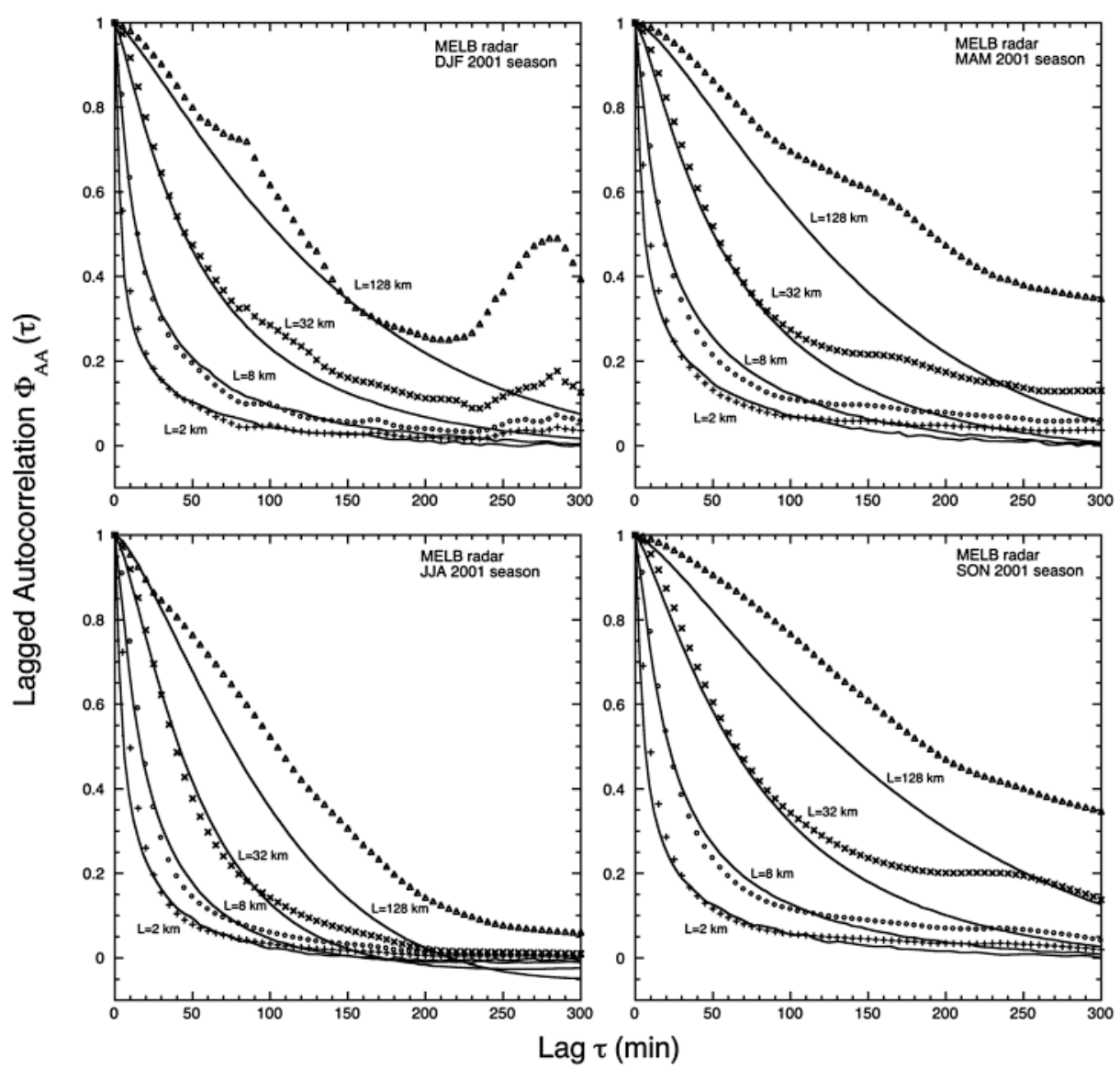

Figure 5. Comparison of the lagged autocorrelation at four different spatial scales $(L=2,8,32$, and $128 \mathrm{~km}$ ) as a function of the separation $s$ as estimated from the MELB radar data (the symbols) and the spectral model (the solid curve) with the parameters listed in Table 1 for the same period as in Figure 3.

However, as was noted in BK96, this approach has the disadvantage that the spectral estimates often suffer from distortions at spatial scales comparable to the size of the area considered ( $L=128 \mathrm{~km}$ in our case) that are artifacts of the periodic boundary condition imposed by the analysis methodology. This is an important issue for us since an intended application of the model is to perform a statistical intercomparison of rain rate data from ground radar and a rain gauge network located within the radar FOV. While there are smoothing techniques for the numerically evaluated spectrum that reduce these distortions somewhat [Jenkins and Watts, 1968], it is not clear to what extent the choice of a particular smoothing prescription would affect our results. Moreover, the radar data was not uniformly spaced in time, which would therefore necessitate further interpolation. To circumvent these difficulties we choose to follow the approach adopted in BK96 and determine the parameters by directly fitting the statistics of the area averages to the model predictions. Our fitting process is in effect suitably weighted so that the model faithfully represents the observed statistics at spatiotemporal scales of interest to us.

[37] Our parameter estimation method takes advantage of the mathematical structure of the model and proceeds in two stages. First, the parameters $\gamma_{0}, L_{0}$, and $v$ are estimated from the spatial statistics. The parameters $v$ and $L_{0}$ are obtained from a suitably weighted least squares fit to the spatial correlation function $\Phi_{\text {pix }}(\mathbf{s}) \equiv \Phi_{A A^{\prime}}(s)$ for $2 \mathrm{~km}$ pixels separated by a distance $s=|\mathbf{s}|$. For simplicity, the slight direction dependence of $\Phi_{\text {pix }}(\mathbf{s})$ expected from the model at small separations is neglected. From the gridded radar images, we first compute the (Pearson) sample correlation coefficients $\rho_{m}$ for all pairs of pixels with spatial separation vectors $\mathbf{s}_{m}$ of length $s_{m}(m=1,2, \ldots, M)$ as the estimate of the theoretical spatial correlation $\Phi_{\text {pix }}\left(\mathbf{s}_{m}\right)$ for a set of $M=66$ selected values of $s_{m}$ ranging from the minimum value of $2 \mathrm{~km}$ up to about $160 \mathrm{~km}$ (slightly less than the length of the diagonal of a $128 \mathrm{~km}$ box). The vectors $s_{m}$ are chosen so that all directions are more or less uniformly represented and there are a substantial number (in the range 200-23,000) of pairs $N_{m}$. We then seek to obtain estimates of $v$ and $L_{0}$ by minimizing the quantity

$$
J\left(v, L_{0}\right)=\sum_{m=1}^{M} w_{m}\left[\rho_{m}-\Phi_{\text {pix }}\left(\mathbf{s}_{m}\right)\right]^{2},
$$

where $w_{m}$ are a set of weights inversely proportional to the variances $\Sigma_{m}{ }^{2}$ of the sample spatial correlation $\rho_{m}$. If the samples entering into the computation of $\rho_{m}$ are independent, then $\Sigma_{m}{ }^{2} \propto 1 / N_{m}$ and consequently the weights are expected to be proportional to the number of samples, i.e., $w_{m} \propto N_{m}$. In reality, there is data dependency caused by the space-time correlation of the rain field, thus reducing the effective number of independent samples. The overall effect of this dependency for our estimation problem is somewhat difficult to assess and will not be taken into consideration here. Evaluation of the model spatial correlations $\Phi_{\text {pix }}\left(\mathbf{s}_{m}\right)$ is carried out from 


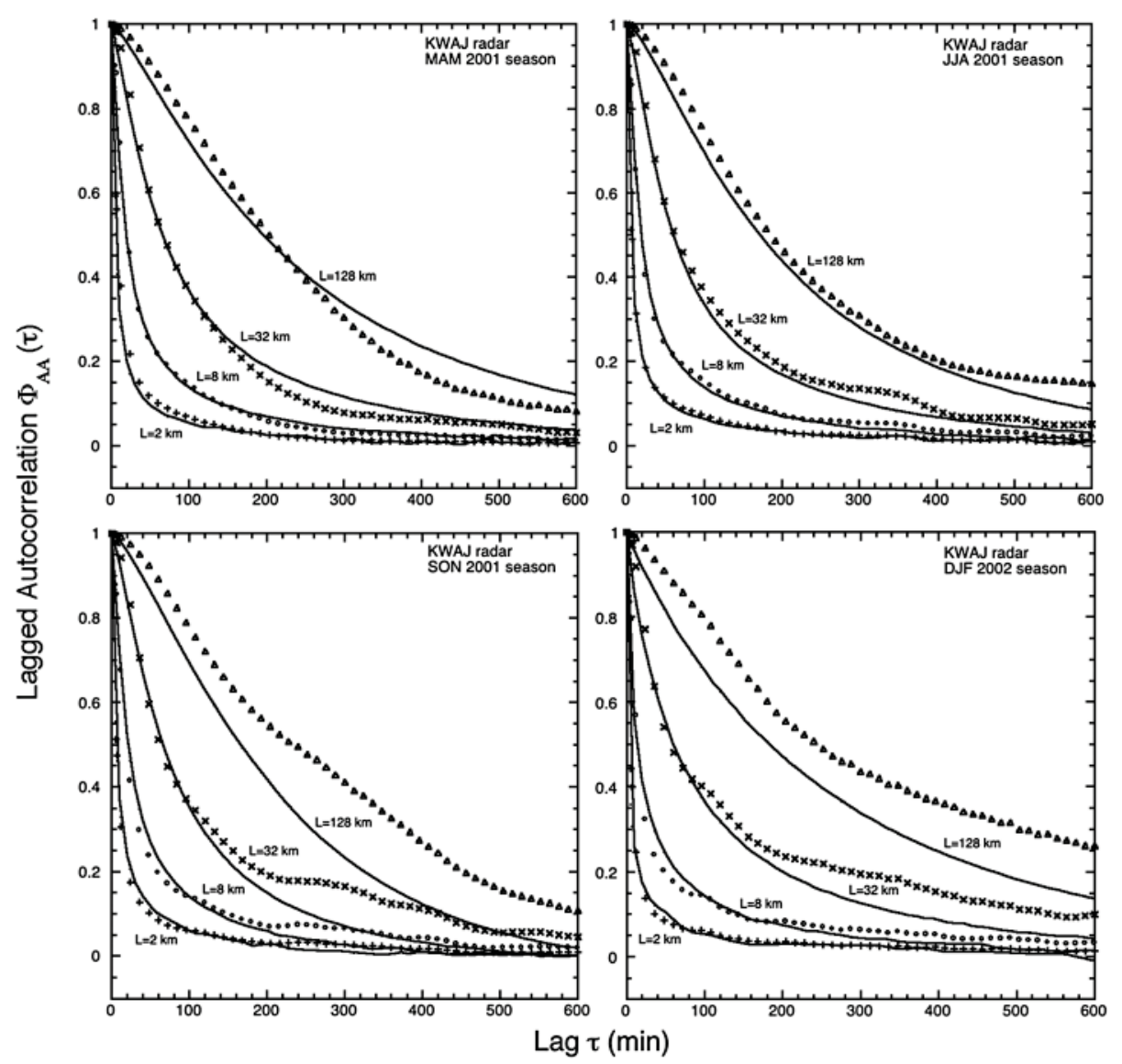

Figure 6. Same as in Figure 5 but for the KWAJ radar data (the symbols) and the spectral model (the solid curve) for the same period as in Figure 4.

equation (B5) using the software Mathematica, version 9, from Wolfram, Inc. The minimum of $J\left(v, L_{0}\right)$ is sought by employing the numerical implementation of the NelderMead Simplex Algorithm [Nelder and Mead, 1965; Press et al, 1992; Lagarias et al, 1998] in Mathematica. A starting value for the index $v$ can be obtained by fitting the asymptotic form equation (B22) to the variance $\sigma_{A}^{2}$ for small $L$. The computation of the spatial parameters $v$ and $L_{0}$ for each data set was quite efficient and took only a few minutes on a desktop computer equipped with two Quad-core Intel Xeon processors. The normalization constant $\gamma_{0}$ is fixed by a least squares fit of the model prediction (using the exact formula (B7)) to the observed variances $\sigma_{A}^{2}$. The Nelder-Mead Algorithm, despite its heuristic nature, is our preferred choice for an optimization method primarily because of its simplicity and speed of execution. For a test run on a KWAJ radar data set, the Differential Evolution Method [Storn and Price, 1997], a global optimization method available within Mathematica, gave results for $v$ and $L_{0}$ that are substantially identical with those from the Nelder-Mead Method but took much longer (about 28 times) to converge.

[38] The next step is to estimate the temporal parameters $\tau_{0}, \beta$ (and therefore $\alpha$ ). They can be determined by fitting the lagged autocorrelation function $\Phi_{A A}(\tau)$ for a particular box size $L$. We calculated the model values $\Phi_{A A}\left(\tau_{j}\right)$ using equations (B6) and (B7) and fit them to the observed values $\hat{\rho}_{j}$ for a $16 \mathrm{~km}$ box and a set of time lags $\tau=\tau_{j}(j=1,2, \ldots, n)$ up to a certain reasonably large $\tau$. Values of $\beta$ and $\tau_{0}$ are estimated by minimizing the quantity

$$
\hat{J}\left(\beta, \tau_{0}\right)=\sum_{j=1}^{n} \hat{w}_{j}\left[\hat{\rho}_{j}-\Phi_{A A}\left(\tau_{j}\right)\right]^{2}
$$

using the Nelder-Mead Simplex Algorithm in Mathematica. The weights $\hat{w}_{j}$ can now be taken to be 1 (the ordinary least squares case) since the numbers of samples are roughly equal for all lags in the range considered. The observed lagged autocorrelation was found to exhibit large systematic departure from the model-predicted form for larger values of $\tau$. We therefore had to restrict the range of $\tau$ over which the fit was carried out (about $400 \mathrm{~min}$ for KWAJ and $200 \mathrm{~min}$ for MELB).

[39] Unfortunately, evaluation of the quantities $\Phi_{A A}\left(\tau_{j}\right)$ involves computing $(2+1)$ dimensional integrals in the Fourier space of $\mathbf{k}$ and $\omega$ making the optimization problem computationally rather onerous. The Nelder-Mead Algorithm took a long time to converge. The execution time depended on the number of lag values employed to define the sum of squares $\hat{J}\left(\beta, \tau_{0}\right)$; for each data set it typically took about $1 \mathrm{~h}$ per value of $j$ on a workstation equipped with an Intel i7 870 processor. We should note that in estimating these parameters, the short distance cut-off parameter $\Lambda$ is implicitly set to zero with the anticipation that it is sufficiently small compared to the smallest spatial scale accessible to radar measurements. Its 


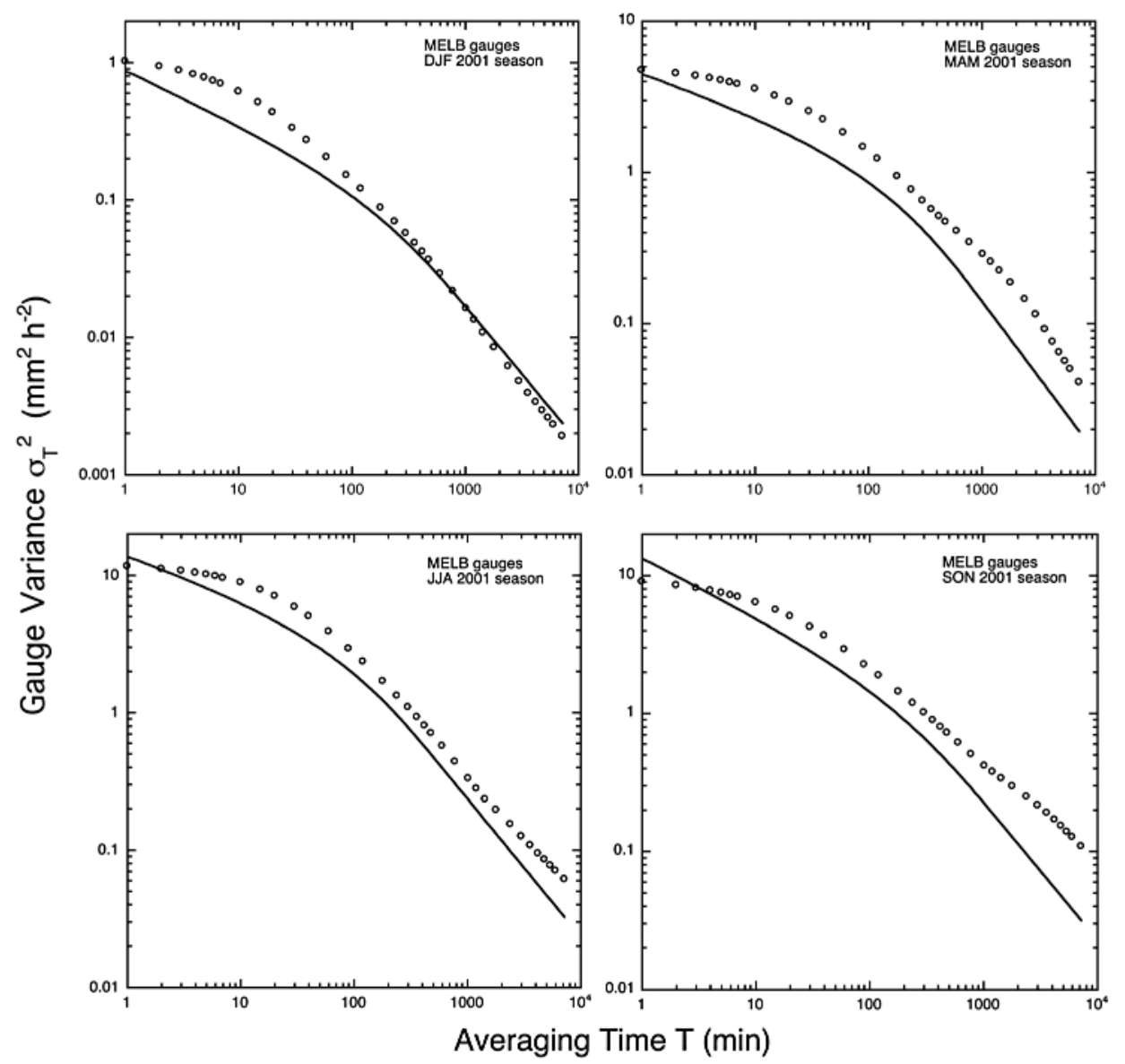

Figure 7. Plot of the variance of time-averaged point rain rate ${\sigma_{T}}_{T}^{2}$ as a function of the averaging scale $T$ for precipitation data from the TB gauges located within the MELB radar FOV. The panels exhibit results for the same time period December 2000 to November 2001 divided into four seasons as in Figure 2. The open circles denote the observed variance and the solid curve represents the model results.

value can only be meaningfully estimated from the gauge data at very short time scales.

[40] We divided the 2A-53 annual radar precipitation data sets from the KWAJ and MELB radars into four 3 month long seasons - Winter (December-February, or DJF), Spring (March-May, or MAM), Summer (June-August, or JJA) and Autumn (September-November, or SON), and carried out the estimation of the model parameters for each. In doing so, we implicitly regard the statistics of each season as stationary in the wide sense. Here we present results of parameter estimation for four seasons for each of these two radars. For the MELB radar, we selected the 2001 DJF, MAM, JJA, and SON seasons. For the KWAJ radar, DJF 2001 data had to be excluded from the analysis because of the highly erratic behavior of the temporal correlation, and so we considered DJF 2002 instead as a representative example.

[41] The model parameters are listed in Table 1. The spatial index $v$ lies in the range -0.27 to -0.33 for the KWAJ radar and -0.11 to -0.22 for the MELB radar. The temporal index $\beta$ is found to be substantially greater than 1 , in fact lying in the narrow range $1.2-1.3$, in all cases except one, namely the KWAJ DJF 2002 season. The characteristic length and time scales $L_{0}$ and $\tau_{0}$ are generally longer for the KWAJ data sets than the MELB data sets, which is presumably attributable to the purely oceanic environment of the former. The constant $\gamma_{0}$ varies widely from one data set to another and appears to be related to the mean rain rate. Figure 2 shows a comparison of the variances $\sigma_{A}^{2}$ deduced from the data (symbols) and those computed from the model (solid curve) for various box sizes $L$. The fits to the spatial correlation function $\Phi_{A A^{\prime}}(s)$ for the $L=2 \mathrm{~km}$ radar pixels as a function of the separation $s$ are shown in Figures 3 and 4 for the MELB and KWAJ radars, respectively. The quality of the fits for the temporal statistics is illustrated in Figure 5 (for the MELB radar) and Figure 6 (for the KWAJ radar) by the plots of $\Phi_{A A}(\tau)$ at several other spatial scales. It is seen that the model fits the observation reasonably well for spatial scales $L$ between 2 and $32 \mathrm{~km}$, but the fit becomes worse at larger scales. By allowing the order of the time derivative $\beta$ to be an adjustable noninteger parameter, a closer fit at small $\tau$ is achieved for the temporal statistics compared to the $\beta=1$ case of the model, as illustrated in Appendix B (Figure B2) for the KWAJ JJA 2001 season.

\subsection{Validating the Model with Rain Gauge Data}

[42] In this section we compare the model predictions for the statistics of time-averaged point rain rate outlined in section 2.4 with the statistics of rain gauge observations. The temporal statistics were obtained from a rain gauge data set (TRMM standard product 2A-56) for the 

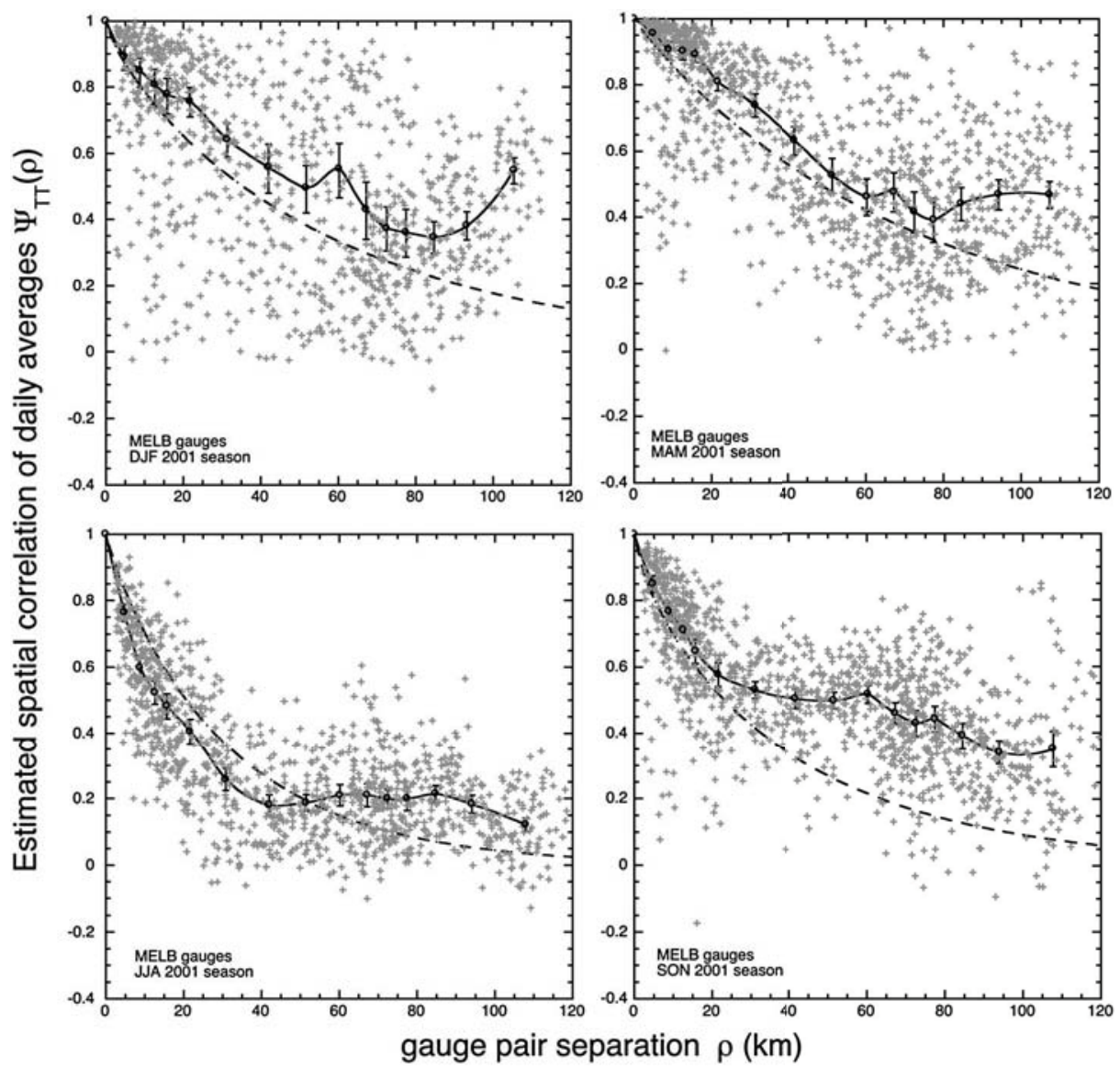

Figure 8. Plot of the spatial correlation $\Psi_{T T}(\rho)$ of the daily averaged gauge data as a function of the gauge separation $\rho$ for the same data set as in Figure 7. The panels exhibit results for the same time period December 2000 to November 2001 divided into four seasons as in Figure 2. The + symbols denote the observed correlation for each gauge pair. The open circles denote the observed correlation averaged over all gauge pairs in each distance bin interpolated by the solid curve. The error bars are estimated according to the method described in the text. The dashed curve represents the results computed from the model.

same time period over which the spectral model parameters were obtained in section 3. The data consists of estimates of 1 min rain rate derived from rainfall accumulations in the tipping bucket (TB) gauges located within the same central $128 \mathrm{~km}$ area within the radar field-of-view (FOV) that was used to extract the radar statistics during a particular period of observation (Figure 1). For the KWAJ radar FOV, there were only seven gauge locations, each equipped with paired TB gauges and, moreover, not all of them were available for every season. For the MELB radar, there were about 50-60 gauges available within the central $128 \mathrm{~km}$ box.

[43] We computed an estimate of the observed temporal variance $\sigma_{T}^{2}$ for $T$ in the range $1-7200 \mathrm{~min}$ by taking the average over a moving window of length $T$ for each gauge along the time series and computing the variance for the full set of gauges for the entire season. The variances for the individual gauges were averaged together to estimate $\sigma_{T}^{2}$. The spatial correlation of the gauge pairs $\Psi_{T T}(\rho)$ was also computed for daily averaged rain rates $(T=1440 \mathrm{~min})$. For the MELB data set, the average spatial correlations between gauge pairs were estimated by binning them into spatial separation bins. The average over each bin was estimated as follows. A Fisher $z$-transform $z=\tanh ^{-1} \Psi$, which maps the interval $(-1,1)$ onto the real line, was applied to the correlation estimates for each gauge pair. It is well-known that [Hawkins, 1989] for a fairly large class of probability distributions of the correlation estimates $\Psi$, the variable $z$ is nearly normal and consequently, the standard estimates of the confidence intervals apply. The inverse transform $\Psi=\tanh z$ is then applied to the statistics obtained from the normal theory to determine the average correlation and the corresponding 95\% confidence intervals for each separation bin. In practice, the histograms of the $z$-transformed values turned out to be slightly non-normal. We therefore checked the accuracy of our results by constructing a bootstrap distribution from 10,000 replicas of a random sample drawn from the underlying empirical distribution and constructing bootstrap confidence intervals [Efron, 1981]. The resulting bias of the bootstrap estimate of the mean was found to be negligibly small and the estimated confidence intervals agreed well with the normal statistics estimates. For the KWAJ data, the gauge distribution was very sparse, and with the small number of rain gauges that were available in the area, 

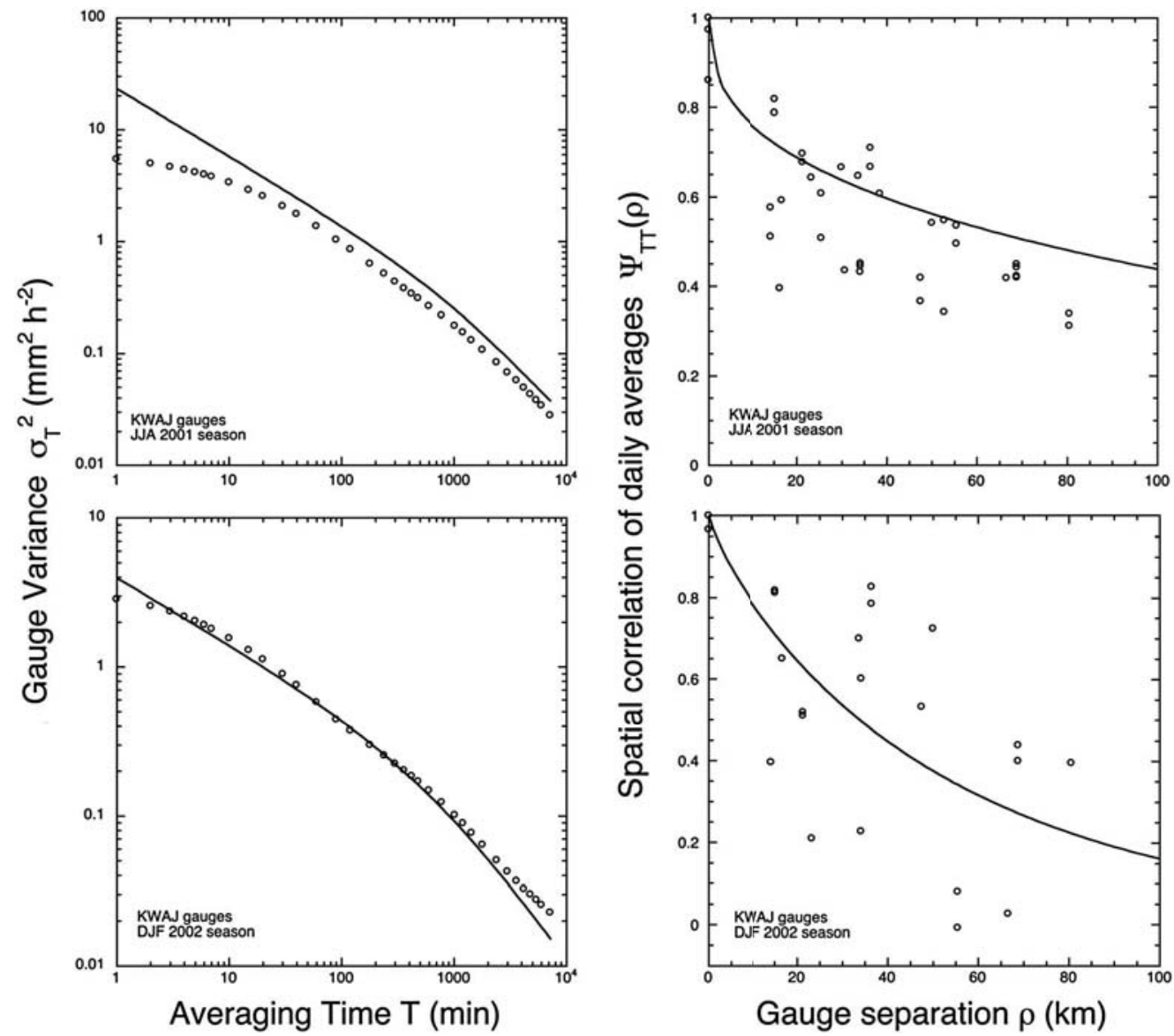

Figure 9. Plot of the (left) variance $\sigma_{T}^{2}$ as a function of the averaging scale $T$ and the (right) spatial correlation $\Psi_{T T}(\rho)$ of the daily averaged gauge data as a function of the gauge separation $\rho$ for the Summer (June-August) 2001 season KWAJ gauge data.

only a few appeared to give reliable results. As a result, each pair was considered individually even though the resulting spatial correlation estimates were much noisier. Moreover, it was decided to leave out of the analysis certain gauges that showed anomalously small cross correlation with their duplicates and other nearby gauges.

[44] It should be noted that the TB estimates at small $T$ (less than about $7-10 \mathrm{~min}$ ) are known to be somewhat unreliable [Habib et al., 2001]. This is because the TB gauge data consist of a sequence of tip times of a bucket of a certain fixed capacity. The interpolation scheme based on a cubic spline algorithm [Wang et al., 2008] used to generate the 1 min time series of rain rates, leads to approximations when isolated single and double tips occur at low rain rates. The statistics of rain rates at small time scales $T$ can thus be potentially distorted by the artifacts of this algorithm.

[45] Using the parameters extracted from the fit to the 2A-53 radar data, we compute the statistics of time-averaged point rain rate from the model using equations (B18) and (B19) and compare them with the corresponding observed quantities computed from the 2A-56 rain gauge data. This comparison provides a powerful validation of the model. Figure 7 exhibits our results for the observed and the model-predicted variance $\sigma_{T}^{2}$ as a function of $T$ for the MELB DJF-SON 2001 data in the range 1-7200 min. A comparison of the corresponding spatial autocorrelation $\Psi_{T T}(\rho)$ of the daily averages as a function of gauge separation $\rho$ for the MELB data is shown in Figure 8 along with a scatter-plot of the correlation for the individual pairs. Figure 9 displays plots for the variance and the spatial correlation for the KWAJ gauges for the JJA 2001 and DJF 2002 seasons as illustration of the kind of results obtained. Since it includes only nine gauges, we display the correlation for the individual pairs without attempting to group them into spatial separation bins. Rough estimates of the point variance $\sigma_{0}^{2}$ and the corresponding values of the spectral cut-off $\Lambda$ inferred from them using equation (34) are also included in the last two columns of Table 1 .

\section{Discussion}

\subsection{Radar Statistics}

[46] As already noted above, the complex behavior of the space-time statistics especially at the larger spatial scales limits the extent to which one can expect quantitative agreement between the radar data and our model. One limiting factor is that the spectral model assumes space-time stationarity of the statistics at the outset. These assumptions greatly facilitate the formulation of a statistical model in a parsimonious manner with a relatively small number of adjustable parameters. However, they also preclude application of the model to physical situations where local meteorological effects can, in general, lead to nonstationary statistics. The assumption of 
spatial isotropy can be relaxed by introducing a form of $\tau_{\mathrm{k}}$ in equation (6) that depends on both the magnitude and direction of $\mathbf{k}$. Such a model will necessarily contain more parameters and will be more difficult to fit to data.

[47] For both the MELB and the KWAJ data sets, the model is able to fit the spatial statistics fairly well at small spatial scales. The observed scatter in the plots of the spatial correlation function $\Phi_{A A^{\prime}}(s)$ at large $s$ (Figures 3 and 4) in part reflects the fluctuation resulting from increasingly smaller sample sizes. However, the model has difficulty fitting the observed spatial correlation at larger separations where a small nonzero tail appears to linger. This is also reflected by the fact that the model prediction for the variance $\sigma_{A}^{2}$ as a function of box size $L$ in general agrees well with the observed value for spatial scales up $32 \mathrm{~km}$ but falls short at the larger scales (see Figure 2). This is appropriate for an application of the model to problems that involve statistical comparisons between precipitation data from ground radar and a dense network of rain gauges located within the radar FOV. This is in contrast to BK96 where the fitting process was weighted so as to fit the larger scales better, since there, the intended application was to faithfully capture the statistics of large-area averages measured from satellites.

[48] However, fitting the temporal statistics proved to be a rather challenging task. Quality of the fit for the temporal autocorrelation varies from one data set to another. Overall, the fit was better for the KWAJ data set than the MELB data set. The observed $\Phi_{A A}(\tau)$ often deviates from the model behavior at large spatial and temporal scales as evidenced by the presence of heavy tails in Figures 5 and 6 . Consequently, the fit had to be restricted to a limited range of space-time scales.

[49] For the MELB radar, the complication arises in part due to the fact that it is located close to the Atlantic coast, and the $128 \mathrm{~km}$ box centered at the radar lies partly over land and partly over the open ocean. Thus, strictly speaking, the key assumption of spatiotemporally stationary statistics, which is at the heart of the theoretical foundation of the model, is violated here. This probably explains why the MELB data fits are in general the poorer of the two radar data sets. We have already noted that the lagged autocorrelation $\Phi_{A A}(\tau)$ exhibits oscillatory tail behavior at large lags that become more pronounced for the larger spatial averaging scales. Our spectral model also predicts a similar behavior in the case $\beta>1$ (see Figure B1) that is, however, too small to match the observations. This makes it rather difficult to find a set of temporal parameters that fit $\Phi_{A A}(\tau)$ uniformly well over the entire range of scales. Although we are unable to fully explain this discrepancy, we suspect that it is partly attributable to the interaction between a strong local diurnal cycle and the land-sea contrast in the coastal region. Because of these oscillations, it is difficult to obtain a stable estimate of the integral correlation time $\tau_{A}$ from the data that could be reliably compared with the model prediction, as was done in $\mathrm{KB} 03$ for radar rainfall data over the open oceans to fit the model in the $\beta=1$ case. For the KWAJ radar, the purely oceanic environment makes the assumption of spatial homogeneity a reasonable one. However, deviation from temporally stationary behavior is fairly common even in an oceanic environment. This generally manifests itself in the form of a time-dependent mean and variance. In this case different subsets of a season, say different months, will have unequal rain rate variance. Fitting the model to data that is inherently heteroscedastic forces one to restrict the space-time size of the sample. Thus, there is an inevitable compromise: too large a sample may strongly violate the model assumption of space-time stationarity, but too small a sample may yield noisy and unreliable statistics. Partitioning the data into seasons is merely a practical way to cope with the situation, the working assumption being that the statistics remain stationary in the course of a particular season. Seasonal dependence of the model parameters accounts for variation of the statistics from one season to another. This may not always work as the KWAJ DJF 2001 data illustrates, where isolated events cause substantial deviation from stationarity. Because of temporally nonstationary behavior, the statistics can defy description in terms of a spectral model like one described in this paper. The DJF 2002 results presented here were relatively well-behaved. Nonstationary statistics caused by local meteorological effects, such as land-sea contrast and orography, are rather difficult to quantify. A model like ours has no effective way of dealing with it and thus the prospect of a good agreement is somewhat tempered in many cases.

[50] Clearly, the radar data does not currently have adequate spatial resolution to be able to reveal substantial departure from the power law singular nature of the spatial statistics predicted by the model. In fact, the singularity of $\sigma_{A}^{2}$ is weak enough to be still consistent with its observed growth rate at the smallest length scales probed by the radar. Consequently, there is no need for postulating the spectral cut-off $\Lambda$ discussed in section 2.5 purely on the basis of the radar statistics alone. However, the need for a small distance cut-off becomes apparent when one considers the statistics of gauge data, which can access a broader range of space-time scales.

\subsection{Gauge Statistics}

[51] Wang et al [2008] recommend that while comparing the model prediction with the observed gauge statistics, one should exclude the values of time averaging scale $T$ less than 4-7 min so that the TB gauge estimates can be considered to be reasonably accurate. In Figure 7 we note that the model substantially underestimates (by a factor of about 1.5 to 2) the observed values of $\sigma_{T}^{2}$ obtained by averaging over all the available gauges in the MELB radar FOV. In Figure 8 the spatial correlation $\Psi_{T T}(\rho)$ for individual gauge pairs exhibits a rather large scatter for all the four 2001 seasons examined, perhaps indicating substantial spatial anisotropy of the rain statistics in the MELB radar area. The model reproduces the binned average of the observed values of $\Psi_{T T}(\rho)$ reasonably well for small separations. The anomalous behavior of the observed values appearing at longer distance scales is presumably caused by large-scale phenomena similar to those in the radar data, and like the latter, it is difficult to associate it with local meteorology. The large scatter in the values of the spatial correlation for the individual gauge pairs in the vicinity of the MELB radar (Figure 8) is similar to what was seen in [Ciach et al., 1997] for daily averages near Darwin, Australia. Agreement between the observed and the model-generated statistics for the gauges is considered adequate given the limitation that the gauges only sample the land portion of the $128 \mathrm{~km}$ box. For the KWAJ data (Figure 9), far fewer data points are available, but the situation appears to be generally similar. The observed values of $\sigma_{T}^{2}$ agree fairly well with the model prediction, but the extent of agreement is less clear for the spatial correlation $\Psi_{T T}(\rho)$. 
[52] For averaging time scales $T$ less than about $30 \mathrm{~min}$, the observed $\sigma_{T}^{2}$ does not increase as steeply as predicted by the spectral model, for which the asymptotic behavior (B26) suggests a power law divergence. Rather, it appears to flatten out to a finite value $\sigma_{0}^{2}$ as we consider smaller and smaller values of $T$. While this may in part be due to a possible distortion of the TB gauge statistics at small $T$, we suspect that this effect alone is not enough to account for the observed modified behavior. In fact a similar flattening trend is noticeable also for the variance of the rain rate as measured by an optical disdrometer (courtesy Dr. Ali Tokay, not shown), which does not suffer from the kind of error that plagues the TB estimates. It appears reasonable to conclude that $\sigma_{T}^{2}$ actually tends to a finite value $\sigma_{0}^{2}$ as $T \rightarrow 0$ indicating the need of a short distance cut-off $\Lambda$. As expected, the values of $\Lambda$ inferred from the rough estimates of $\sigma_{0}^{2}$ listed in Table 1 are much smaller than the smallest radar spatial scale, $2 \mathrm{~km}$, thus providing an a posteriori justification for neglecting it during parameter estimation from radar data.

\section{Summary and Conclusion}

[53] In this paper we have presented a spectral model of precipitation that describes the second moment statistics of rain rate from both radar and rain gauge data within a unified framework. The model is based on a linear stochastic differential equation involving a fractional-order time derivative that generalizes a simpler predecessor based on an equation that was first order in time. The parameters of the model spectrum are estimated by fitting the radar data. Our parameter estimation can be thought of as being a "skewed fit" since the optimization procedure we adopted is designed to better estimate the smaller scale property of the precipitation field. With two adjustable spectral indices, the model fits the observed statistics of radar rainfall fairly well at smaller spatial and temporal scales. The model is tested by comparing its predictions against the observed statistics of rain rate data taken from a set of gauges located within the radar FOV. With the radar parameters, the model appears to reproduce the scale dependence of the gauge statistics reasonably well. The difficulties experienced by the model at larger scales vary from site to site and from one season to another. They are presumably attributed to the statistically nonstationary nature of the data that is intrinsic to the rainfall phenomenon itself.

[54] The spectral model provides a useful tool for estimation of the sampling error for various radar-gauge intercomparison scenarios [Bell and Kundu, 2003]. Radar at a GV site that is equipped with a dense rain gauge network can be conveniently calibrated using the model as a framework for data fitting. We plan to explore this problem further in a future paper.

\section{Appendix A: Fractional Calculus-A Brief Outline}

[55] The notion of a derivative or integral of fractional order has a long history that is almost as old as calculus itself going back to Leibniz, who mentioned a quantity that in modern terminology would be equivalent to derivative of order $1 / 2$. The concept was first systematically formulated by Liouville with important subsequent contributions by Riemann, Grünwald, Krug, Sonine, Weyl, Riesz, and many other mathematicians. See, e.g., Samko et al. [1993] for a detailed account of the historical developments. For a noninteger order $\beta$ (which can in general even be complex), the usual integral and derivative operators are generalized into one-parameter families of operators ${ }_{a} I_{t}^{\beta}$ and ${ }_{a} D_{t}^{\beta}$ labeled by a real variable $a$, sometimes collectively referred to as fractional differintegral operators [Oldham and Spanier, 2006]. An integral of fractional-order $\alpha$ is defined by the formula

$$
{ }_{a} I_{t}^{\alpha} f(t)=\frac{1}{\Gamma(\alpha)} \int_{a}^{t} \mathrm{~d} u f(u)(t-u)^{\alpha-1}(\alpha>0) .
$$

In the case $\alpha=n>0$, a positive integer, it correctly reduces to

$$
\begin{aligned}
{ }_{a} I_{t}^{n} f(t) & =\int_{a}^{t} \mathrm{~d} u_{1} \int_{a}^{u_{1}} \mathrm{~d} u_{2} \ldots \int_{a}^{u_{n-1}} \mathrm{~d} u_{n} f\left(u_{n}\right) \\
& =\frac{1}{(n-1) !} \int_{a}^{t} \mathrm{~d} u f(u)(t-u)^{n-1}
\end{aligned}
$$

which is the unique solution to the differential equation

$$
y^{(n)}(t)=f(t), y(a)=y^{\prime}(a)=y^{\prime \prime}(a)=\ldots=y^{(n-1)}(a)=0 .
$$

The derivative operator ${ }_{a} D_{t}^{\beta}$ is then defined through

$$
{ }_{a} D_{t}^{\beta} f(t)=\left\{\begin{array}{l}
\frac{1}{\Gamma(-\beta)} \int_{a}^{t} \frac{\mathrm{d} u f(u)}{(t-u)^{1+\beta}} ; \operatorname{Re} \beta<0 \\
\left(\frac{\mathrm{d}}{\mathrm{d} t}\right)^{n}{ }_{a}^{n} I_{t}^{n-\beta} f(t) ; n-1<\operatorname{Re} \beta<n, n>0 .
\end{array}\right.
$$

[56] There is a reciprocal relationship between the fractional integral and derivative operators that is suggested by analogy with traditional calculus. It is usual practice to consolidate the notation by writing ${ }_{a} I_{t}^{\beta}={ }_{a} D_{t}^{-\beta}$ thus formally extending the fractional derivative operators to all orders in a way that clarifies the inverse relation between the two sets of operators introduced separately.

[57] We now list some of the basic properties:

$$
\begin{aligned}
& \text { 1. }{ }_{a} I_{t}^{\alpha} \cdot{ }_{a} I_{t}^{\beta} f(t)={ }_{a} I_{t}^{\alpha+\beta} f(t)={ }_{a} I_{t}^{\beta} \cdot{ }_{a} I_{t}^{\alpha} f(t), \\
& \text { 2. }{ }_{a} I_{t}^{0} f(t)=f(t),{ }_{a} I_{t}^{-n} f(t)=\frac{\mathrm{d}^{n}}{\mathrm{~d} t^{n}} f(t) \equiv f^{(n)}(t), \\
& \text { 3. }{ }_{a} D_{t}^{\alpha} f(t)=\frac{\mathrm{d}}{\mathrm{d} t} I_{t}^{1-\alpha} f(t)={ }_{a} I_{t}^{1-\alpha} f^{\prime}(t)+\frac{f(a)}{\Gamma(1-\alpha)}(t-a)^{-\alpha} .
\end{aligned}
$$

The last relation indicates that the operators $\mathrm{d} / \mathrm{d} t$ and ${ }_{a}$ $I_{t}^{1-\alpha}$ do not commute.

[58] Two cases can be distinguished. In the case when $a$ is finite, one can choose $a=0$ without loss of generality. The derivative operator ${ }_{0} D_{t}^{\beta}$ is the so-called Riemann-Liouville derivative, which acts on classes of functions defined on the half line $[0, \infty)$ and naturally appears in the context of an initial value problem like Brownian motion. The other case arises when one lets $a \rightarrow-\infty$. The derivative ${ }_{-\infty} D_{t}^{\beta}$, which we shall call the Liouville-Weyl derivative, emerges as the relevant fractional derivative operator when one is interested in classes of functions that reside on the entire real line $(-\infty, \infty)$. (Weyl's name is attached to it because he introduced a class of fractional operators that coincides with ${ }_{-\infty} D_{t}^{\beta}$ for the class of periodic functions). Now the initial condition refers to the infinitely distant past and the temporal 


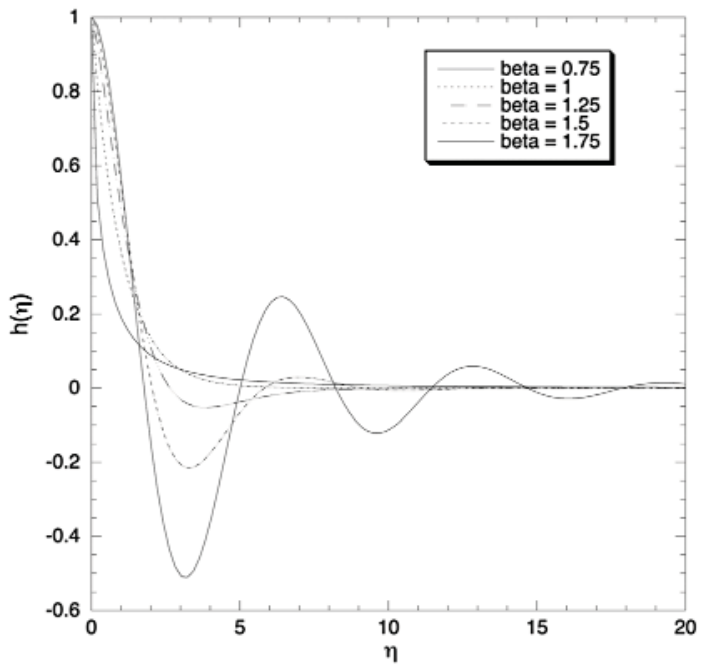

Figure B1. Plot of the function $h(\eta)$ versus $\eta$ for several values of the parameter $\beta$.

evolution is time translation invariant. It is the latter case that is of greater interest to us, since it allows one to describe temporally stationary stochastic processes.

[59] The Liouville-Weyl derivative has the important property that under the operation of Fourier transform defined by the representation

$$
a(\mathbf{k}, t)=(2 \pi)^{-1 / 2} \int_{-\infty}^{\infty} \mathrm{d} \omega e^{-i \omega t} a(\mathbf{k}, \omega),
$$

the usual correspondence $\mathrm{d} / \mathrm{d} t \Leftrightarrow-i \omega$ for ordinary derivatives holds for the fractional derivative as well; that is to say, if $f(t)$ and $F(\omega)$ are Fourier transforms of each other, then ${ }_{-\infty} D_{t}^{\beta} f(t)$ and $(-i \omega)^{\beta} F(\omega)$ are Fourier transforms of each other as well.

\section{Appendix B: Derivation of Some Spectral Model Formulas}

[60] In this Appendix we describe some of the details of the analytical steps in the derivation of the various covariance statistics of precipitation data that are derived from our fractional dynamics model. Most of the steps are very similar to those in the $\beta=1$ case of the present model described in BK96 and KB03 and we will be brief about them.

[61] First, we examine the lagged covariance of the Fourier components of the precipitation field $c(k, \tau)$ given by equation (13), which is the basic building block of the various temporal statistics of interest. The function $h(\eta)$ is defined by the integral representation

$$
h(\eta)=\left(\frac{2}{\pi}\right)^{1 / 2} \frac{1}{g(\beta)} \int_{0}^{\infty} \mathrm{d} \xi \frac{\cos (\xi \eta)}{\xi^{2 \beta}+2 \cos (\beta \pi / 2) \xi^{\beta}+1}
$$

with $h(0)=1$. An explicit formula for the normalization factor $g(\beta)$ is obtained by setting $\eta=0$ and evaluating the $\xi$-integral. When $-2<\beta<2$, it has the explicit form

$$
g(\beta)=-\frac{\sqrt{2 \pi}}{\beta} \frac{\cot (\beta \pi / 2)}{\sin (\pi / \beta)},
$$

which can be shown to be positive despite its appearance.
Plots of $h(\eta)$ are shown in Figure B1 for several values of $\beta$ between $1 / 2$ and 2 . The case $\beta=1$ is elementary: the function $h(\eta)$ is simply $\exp (-\eta)$. When $\beta<1, h(\eta)$ decreases monotonically and rapidly approaches zero for large $\eta$. When $\beta>1, h(\eta)$ exhibits damped oscillatory behavior around zero as $\eta$ increases.

[62] Next we turn to the statistics of area-averaged rain rate. We start with the definition of the lagged rain rate covariance of two areas

$$
\Gamma_{A A^{\prime}}(\mathbf{s}, \tau)=\left(1 / L^{4}\right) \int_{A} \mathrm{~d}^{2} \mathbf{x} \int_{A^{\prime}} \mathrm{d}^{2} \mathbf{x}^{\prime} c\left(\mathbf{s}+\mathbf{x}^{\prime}-\mathbf{x}, \tau\right),
$$

which can also be expressed in terms of a Fourier integral representation

$\Gamma_{A A^{\prime}}(\mathbf{s}, \tau)=(2 / \pi) \int_{0}^{\infty} \mathrm{d} k_{1} \int_{0}^{\infty} \mathrm{d} k_{2} e^{i \mathbf{k} \cdot \mathbf{s}} \operatorname{sinc}^{2}\left(k_{1} L / 2\right) \operatorname{sinc}^{2}\left(k_{2} L / 2\right) c(k, \tau)$,

where $\operatorname{sinc}(x)=\sin (x) / x$ and $k=\left(k_{1}^{2}+k_{2}^{2}\right)^{1 / 2}$. In the case of zero lag, i.e., $\tau=0$, following the steps detailed in BK96, we obtain the spatial covariance function

$\Gamma_{A A^{\prime}}(\mathbf{s}, 0)=\gamma_{0} \int_{-1}^{1} \mathrm{~d} \xi_{1} \int_{-1}^{1} \mathrm{~d} \xi_{2}\left(1-\left|\xi_{1}\right|\right)\left(1-\left|\xi_{2}\right|\right) C_{v}\left(|\xi+\mathbf{s} / L|\left(L / L_{0}\right)\right)$.

For zero separation $(\mathbf{s}=0)$, we get the temporal autocovariance of an area $A$ :

$\Gamma_{A A}(0, \tau)=(2 / \pi) \int_{0}^{\infty} \mathrm{d} k_{1} \int_{0}^{\infty} \mathrm{d} k_{2} \operatorname{sinc}^{2}\left(k_{1} L / 2\right) \operatorname{sinc}^{2}\left(k_{2} L / 2\right) c(k, \tau)$.

Figure B2 illustrates the improvement in the fit to the lagged autocorrelation function $\Phi_{A A}(0, \tau)$ over the $\beta=1$ case in BK96 achieved by letting $\beta$ be an adjustable parameter.

[63] The variance of area-averaged rain rate $\Gamma_{A A}(0,0)$ $=\sigma_{A}^{2}$ can be expressed in two alternative ways, as a spatial integral obtained by setting the separation $\mathbf{s}=0$, in equation (B3) and as a Fourier integral by setting $\tau=0$ in equation (B4). The first approach yields the formula

$$
\sigma_{A}^{2} \equiv\left\langle r_{A}^{\prime 2}\right\rangle=4 \gamma_{0} G\left(v ; L / L_{0}\right)
$$

where we have defined the function

$$
G(v ; z)=\int_{0}^{1} \mathrm{~d} \xi_{1} \int_{0}^{1} \mathrm{~d} \xi_{2}\left(1-\xi_{1}\right)\left(1-\xi_{2}\right) C_{v}\left(z\left(\sqrt{\xi_{1}^{2}+\xi_{2}^{2}}\right)\right) .
$$

As pointed out in KB03, upon equating the two formulas in the case $\beta=1$, one obtains the useful identity:

$$
\int_{0}^{\infty} \mathrm{d} \kappa_{1} \int_{0}^{\infty} \mathrm{d} \kappa_{2} I\left(\kappa_{1}, \kappa_{2} ; L / L_{0}, \lambda\right)=\frac{2 \pi}{\Gamma(1+\lambda)} G\left(\lambda ; L / L_{0}\right)
$$

where, for brevity, we have defined

$$
I\left(\kappa_{1}, \kappa_{2} ; L / L_{0}, \lambda\right)=\frac{\operatorname{sinc}^{2}\left(\kappa_{1} L / 2 L_{0}\right) \operatorname{sinc}^{2}\left(\kappa_{2} L / 2 L_{0}\right)}{\left[1+\kappa_{1}^{2}+\kappa_{2}^{2}\right]^{1+\lambda}} .
$$




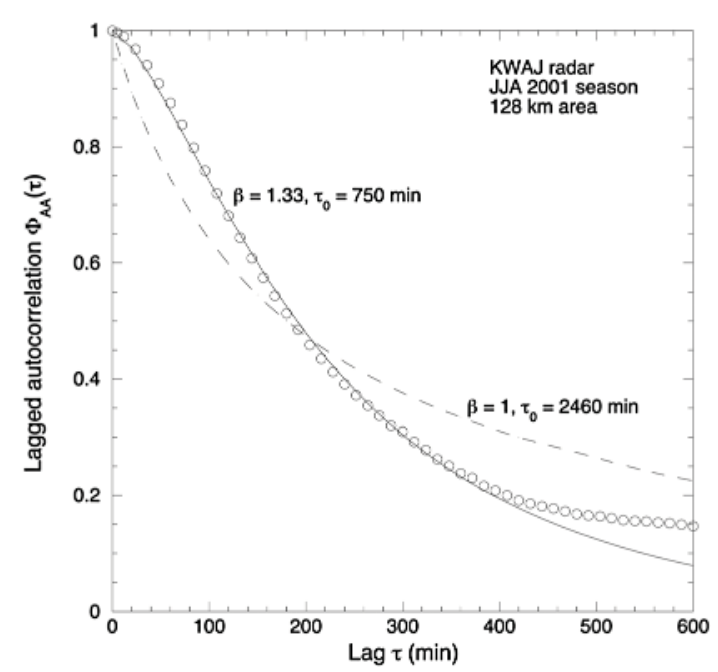

Figure B2. Sample plots of the observed temporal autocorrelation $\Phi_{A A}(\tau)$ for a $128 \mathrm{~km}$ box (open circles) compared with its model prediction corresponding to the best fit values of $\beta$ and $\tau_{0}\left(\beta=1.33, \tau_{0}=750 \mathrm{~min}\right)$ for the same spatial scale (solid curve) and in the case $\beta=1$ with best fit value $\tau_{0}=2460$ min (dashed curve).

[64] Next we evaluate the characteristic time scale $\tau_{A}$ defined by equation (23) as follows. In KB03 the following identity was proved:

$$
\tau_{A} \sigma_{A}^{2}=\sqrt{\pi / 2} \widetilde{\Gamma}_{A A}(\omega=0),
$$

where $\widetilde{\Gamma}_{A A}(\omega)$ is the Fourier transform of $\Gamma_{A A}(0, \tau)$. In view of the Fourier representation (B6), $\widetilde{\Gamma}_{A A}(\omega)$ can be expressed in a similar form

$$
\widetilde{\Gamma}_{A A}(\omega)=(2 / \pi) \int_{0}^{\infty} \mathrm{d} k_{1} \int_{0}^{\infty} \mathrm{d} k_{2} \operatorname{sinc}^{2}\left(k_{1} L / 2\right) \operatorname{sinc}^{2}\left(k_{2} L / 2\right) S(k, \omega),
$$

where $S(k, \omega)$ is the model spectrum. Evaluating the right hand side of (B11) with the explicit form of the spectrum and using the relation (18) between the constants $F_{0}$ and $\gamma_{0}$, we get

$$
\widetilde{\Gamma}_{A A}(\omega=0)=\frac{2 \gamma_{0} \Gamma(1+v)}{\pi g(\beta)} \int_{0}^{\infty} \mathrm{d} \kappa_{1} \int_{0}^{\infty} \mathrm{d} \kappa_{2} I\left(\kappa_{1}, \kappa_{2} ; L / L_{0}, \alpha \beta-1\right) .
$$

With the help of the identity (B9) this can be recast in the form

$$
\widetilde{\Gamma}_{A A}(\omega=0)=\frac{4 \gamma_{0} \tau_{0} \Gamma(1+v)}{\Gamma\left(2+2 v^{\prime}\right) g(\beta)} G\left(1+2 v^{\prime} ; L / L_{0}\right) .
$$

Then equation (B11) yields

$$
\tau_{A} \sigma_{A}^{2}=\frac{2 \sqrt{2 \pi} \gamma_{0} \tau_{0} \Gamma(1+v)}{\Gamma\left(2+2 v^{\prime}\right) g(\beta)} G\left(1+2 v^{\prime} ; L / L_{0}\right),
$$

where we have now introduced a new parameter $v^{\prime}$ through the relation

$$
\alpha \beta=2\left(1+v^{\prime}\right)
$$

Note that $v^{\prime}=v$ in the "old" spectral model with $\beta=1$. From equations (16) and (B14) we have the general relation

$$
2-\frac{1}{\beta}=\frac{1+v}{1+v^{\prime}} .
$$

From equation (B15) it follows immediately that $v^{\prime}>v$ and $v^{\prime}<v v$ correspond respectively to the cases $\beta>1$ and $\beta<1$. Combining equation (B13) and the expression (B7) for $\sigma_{A}^{2}$, we get an explicit formula for $\tau_{A}$ :

$$
\tau_{A}=\sqrt{\frac{\pi}{2}} \frac{\tau_{0}}{g(\beta)} \frac{\Gamma(1+v)}{\Gamma\left(2+2 v^{\prime}\right)} \frac{G\left(1+2 v^{\prime} ; L / L_{0}\right)}{G\left(v ; L / L_{0}\right)} .
$$

[65] Next we outline the computation of the temporal statistics $\sigma_{T}^{2}$ and $\Psi_{T T}(\rho)$ needed to describe the gauge data. The space-time covariance of rain rate averaged over two time intervals of length $T$ lagged by time $\tau$ measured at two points $\mathbf{x}$ and $\mathbf{x}^{\prime}$ separated by a distance $\rho$, namely,

$$
\begin{aligned}
\Gamma_{T T^{\prime}}(\rho, \tau) & \equiv\left\langle(1 / T) \int_{0}^{T} \mathrm{~d} t R^{\prime}(\mathbf{x}, t) \cdot(1 / T) \int_{0}^{T} \mathrm{~d} t^{\prime} R^{\prime}\left(\mathbf{x}^{\prime}, t^{\prime}+\tau\right)\right\rangle \\
& =\left(1 / T^{2}\right) \int_{0}^{T} \mathrm{~d} t \int_{0}^{T} \mathrm{~d} t^{\prime} c\left(\rho, t-t^{\prime}+\tau\right)
\end{aligned}
$$

can be evaluated as follows. First, as usual, we convert the double integral into a single integral yielding the expression

$$
\begin{aligned}
\Gamma_{T T^{\prime}}(\rho, \tau) & =(1 / T) \int_{-T}^{T} \mathrm{~d} \tau^{\prime}\left(1-\frac{\left|\tau^{\prime}\right|}{T}\right) c\left(\rho, \tau^{\prime}-\tau\right) \\
& =(1 / T) \int_{0}^{T} \mathrm{~d} \tau^{\prime}\left(1-\frac{\tau^{\prime}}{T}\right)\left[c\left(\rho, \tau^{\prime}-\tau\right)+c\left(\rho, \tau^{\prime}+\tau\right)\right]
\end{aligned}
$$

Next we introduce the space-time Fourier transform representation of $c(\rho, \tau)$ via equation (11). After performing the $\tau^{\prime}$-integration explicitly and some further simplification, we obtain the formula

$\Gamma_{T T^{\prime}}(\rho, \tau)=\sqrt{2 / \pi} \int_{0}^{\infty} \mathrm{d} \omega \cos (\omega \tau) \operatorname{sinc}^{2}(\omega T / 2) \int_{0}^{\infty} \mathrm{d} k k J_{0}(k \rho) S(k, \omega)$.

At zero lag this reduces to the spatial covariance of time-averaged rain rate:

$$
\Gamma_{T T}(\rho, 0)=\sqrt{2 / \pi} \int_{0}^{\infty} \mathrm{d} \omega \operatorname{sinc}^{2}(\omega T / 2) \int_{0}^{\infty} \mathrm{d} k k J_{0}(k \rho) S(k, \omega) .
$$

The variance $\sigma_{T}^{2}$ is obtained by specializing equation (B18) to the case $\rho=0$ :

$$
\sigma_{T}^{2}=\sqrt{2 / \pi} \int_{0}^{\infty} \mathrm{d} \omega \operatorname{sinc}^{2}(\omega T / 2) \int_{0}^{\infty} \mathrm{d} k k S(k, \omega) .
$$

[66] Finally, we investigate the asymptotic behavior of the area and time-averaged statistics by isolating the leading singularity in the area/time integrals in which $c(\rho, \tau)$ is replaced by its scaling approximation $c^{(\infty)}(\rho, \tau)$. It is convenient to express the singular term in terms of the dimensionless variables 


\section{KUNDU AND TRAVIS: A SPECTRAL MODEL OF RAIN STATISTICS}

$\rho *, \tau *, L *=L / L_{0}$ and $T_{*}=T / \tau_{0}$. In the scaling limit $\tau_{*}, L * \rightarrow 0$, the lagged autocovariance of area-averaged rain rate has the singularity structure

$$
\Gamma_{A A}(0, \tau) \sim \gamma_{0} L_{*}^{-2|v|} \varphi_{1}\left(\tau_{*} / L_{*}^{\alpha} ; \alpha, \beta\right),
$$

where the scaling function $\varphi_{1}(u ; \alpha, \beta)$ is defined as

$\varphi_{1}(u ; \alpha, \beta)=4 \int_{0}^{1} \int_{0}^{1} \mathrm{~d} z_{1} \mathrm{~d} z_{2}\left(1-z_{1}\right)\left(1-z_{2}\right)\left(z_{1}^{2}+z_{2}^{2}\right)^{-|v|} \varphi\left(u\left(z_{1}^{2}+z_{2}^{2}\right)^{-\alpha / 2} ; \alpha, \beta\right)$.

In particular, as was shown in $\mathrm{KB} 03$, the variance $\sigma_{A}^{2} \equiv$ $\Gamma_{A A}(0,0)$ has the limiting behavior

$$
\sigma_{A}^{2} \sim A+B L_{*}^{-2|v|}
$$

plus terms that vanish as $L_{*} \rightarrow 0$. This result continues to hold in the more general $\beta \neq 1$ case, where $A$ and $B$ are constants given by

$$
\begin{aligned}
& A=\frac{1}{2} \gamma_{0} \Gamma(-|v|), \\
& B=2^{1+2|v|} \gamma_{0} \Gamma(|v|) \int_{0}^{1} \mathrm{~d} \xi_{1} \int_{0}^{1} \mathrm{~d} \xi_{2}\left(1-\xi_{1}\right)\left(1-\xi_{2}\right)\left(\xi_{1}^{2}+\xi_{2}^{2}\right)^{v} .
\end{aligned}
$$

[67] The integral correlation timescale $\tau_{A}$ given by equation (B12) has the power law dependence $\tau_{A} \sim L_{*}^{2|v|}$ as $L_{*} \rightarrow 0$. Similarly, in the scaling limit $\rho_{*}, T_{*} \rightarrow 0$ the spatial covariance of time-averaged rain rate has the singularity structure

$$
\Gamma_{T T}(\rho, 0) \sim \gamma_{0} \rho_{*}^{-2|v|} \varphi_{2}\left(T_{*} / \rho_{*}^{\alpha} ; \alpha, \beta\right)
$$

where we have introduced a second scaling function

$$
\varphi_{2}(s ; \alpha, \beta)=2 \int_{0}^{1} \mathrm{~d} z(1-z) \varphi(z s ; \alpha, \beta) .
$$

The asymptotic behavior of the variance of time-averaged rain rate $\sigma_{T}^{2} \equiv \Gamma_{T T}(0,0)$ for small $T *$ is inferred from equations (B24) and (B25) by making use of the limit (32):

$$
\sigma_{T}^{2} \sim(1-|v| / \alpha)^{-1}(1-2|v| / \alpha)^{-1} \gamma_{0} K T_{*}^{-2|v| / \alpha}
$$

[68] Acknowledgments. We thank David Marks of Science Systems Applications International, Inc. and NASA/GSFC for in-depth discussions on various details regarding the TRMM GV data used in this study and for providing us with Figure 1. We are also grateful to Bikas K. Sinha of Department of Mathematics and Statistics, UMBC for his careful reading of the paper and Anindya Roy also of Department of Mathematics and Statistics, UMBC for many helpful discussions. Comments and constructive criticisms from the associate editor and two other anonymous reviewers on various aspects of the work, especially the parameter estimation method, greatly helped to improve the presentation of the paper. The research conducted in this paper was supported by the GPM (Global Precipitation Measurement) GV group as a part of the NASA Precipitation Measurement Missions (PMM) program. One of us (J.E.T.) gratefully acknowledges financial support from a JCET Fellowship.

\section{References}

Bell, T. L. (1987), A space-time stochastic model of rainfall for satellite remote sensing studies, J. Geophys. Res., 92, 9631-9643, doi:10.1029/ JD092iD08p09631.

Bell, T. L., and P. K. Kundu (1996), A study of sampling error in satellite rainfall estimates using optimal averaging of data and a stochastic model, J. Climate, 9, 1251-1268, doi:10.1175/1520-0442(1996)009<1251: ASOTE $>2.0 \mathrm{CO} ; 2$.

Bell, T. L., and P. K. Kundu (2003), Comparing satellite rainfall estimates with rain gauge data: Optimal strategies suggested by a spectral model, J. Geophys. Res., 108(D3), 4121, doi:10.1029//2002JD002641.

Beran, J. (1994), Statistics of Long-Memory Processes, Monographs on Statistics and Probability 61, Chapman and Hall, New York.

Ciach, G. J., W. F. Krajewski, E. N. Anagnostou, M. L. Baeck, J. A. Smith, J. R. McCollum, and A. Kruger (1997), Radar rainfall estimation for ground validation studies of the Tropical Rainfall Measuring Mission, J. Appl. Meteorol., 36, 735-747, doi:10.1175/1520-0450-36.6.735.

Efron, B. (1981), Nonparametric standard errors and confidence intervals (with discussion), Can. J. Stat., 9, 139-172.

Frisch, U. (1995), Turbulence: The Legacy of A.N. Kolmogorov, Cambridge Univ. Press, Cambridge, U.K.

Habib, E., W. F. Krajewski, and A. Kruger (2001), Sampling errors of tipping-bucket rain gauge measurements, J. Hydrol. Engng., 6(2), 159-166, doi:10.1061/(ASCE)1084-0699(2001)6:2(159).

Hawkins, D. L. (1989), Using U-statistics to derive the asymptotic distribution of Fisher's Z statistic, The American Statistician, 43(4), 235-237, doi: $10.2307 / 2685369$.

Jenkins, G. M., and D. G. Watts (1968), Spectral Analysis and its Applications, Holden-Day, San Francisco, CA.

Kedem, B., and L. S. Chiu (1987), Are rain rate processes self-similar?, Water Resour. Res., 23(10), 1816-1618, doi:10.1073/pnas.84.4.901.

Kundu, P. K., and T. L. Bell (2003), A stochastic model of space-time variability of mesoscale rainfall: Statistics of spatial averages, Water Resour. Res., 39(12), 1328 doi:10.1029/2002WR001802.

Kundu, P. K., and T. L. Bell (2006), Space-time scaling behavior of rain statistics in a stochastic fractional diffusion model, J. Hydrol., 322, 49-58, doi:10.1016/j.jhydrol.2005.02.031.

Kundu, P. K., and R. K. Siddani (2007), A new class of probability distributions for describing the spatial statistics of area-averaged rainfall, J. Geophys. Res., 112, D18113, doi:10.1029/2006JD008042.

Lagarias, J. C., J. A. Reeds, M. H. Wright, and P. E. Wright (1998), Convergence properties of the Nelder-Mead Simplex Method in low dimensions, SIAM J. Optim., 9, 112-147, doi:10.1137/S1052623496303470.

Matérn, B. (1960), Spatial variation: Stochastic models and their application to some problems in forest surveys and other sampling investigations, Medd. fran Statens Skogsforskningsinst., 49, 5, 1-144.

Matérn, B. (1986), Spatial Variation: Lecture Notes in Statistics, vol. 36, Second ed., 151 pp. Springer-Verlag, Berlin and New York.

Miller, K. S., and B. Ross (1993), An Introduction to the Fractional Calculus and Fractional Differential Equations, John Wiley, New York.

Nelder, J. A., and R. Mead (1965), A simplex method for function minimization, The Computer Journal, 7, 308-313.

North, G. R., and S. Nakamoto (1989), Formalism for comparing rain estimation designs, J. Atmos. Oceanic Tech., 6, 985-992, doi:10.1175/15200426(1989) 006<0985:FFCRED > 2.0.CO;2.

Oldham, K. B., and J. Spanier (2006), The Fractional Calculus, Dover Publications, Mineola, New York.

Press, W. H., S. A. Teukolsky, W. T. Vetterling, and B. P. Flannery (1992), Numerical Recipes in FORTRAN: The Art of Scientific Computing, $2^{\text {nd }}$ edition, Cambridge University Press, Cambridge, New York.

Samko, S. G., A. A. Kilbas, and O. I. Marichev (1993), Fractional Integrals and Derivatives, Gordon and Breach Science Publishers, New York.

Storn, R., and K. Price (1997), Differential evolution-A simple and efficient heuristic for global optimization over continuous spaces, J. Global Optimization, 11, 341-359, doi:10.1023/A:1008202821328.

Wang, J., B. L. Fisher, and D. B. Wolff (2008), Estimating rain rates from tipping-bucket rain gauge measurements, J. Atmos. Oceanic Tech., 25, 43, doi:10.1175/2007JTECHA895.1.

West, B. J., M. Bologna, and P. Grigolini (2003), Physics of Fractal Operators, Institute of Nonlinear Science, Springer, New York.

Wolff, D. B., D. A. Marks, E. Amitai, D. S. Silberstein, B. L. Fisher, A. Tokay, J. Wang, and J. L. Pippitt (2005), Ground validation for the Tropical Rainfall Measuring Mission (TRMM), J. Atmos. Oceanic Tech., 22, 365-379, doi:10.1175/JTECH1700.1. 